\title{
Arid1a loss potentiates pancreatic $\beta$-cell regeneration through activation of EGF signaling
}

\section{Authors:}

Cemre Celen ${ }^{1}$, Jen-Chieh Chuang ${ }^{1}$, Shunli Shen ${ }^{1}$, Jordan E. Otto ${ }^{3}$, Clayton K. Collings ${ }^{3}$, Xin Luo $^{1}$, Lin $\mathrm{Li}^{1}$, Yunguan Wang ${ }^{1,2}$, Zixi Wang ${ }^{1}$, Yuemeng Jia ${ }^{1}$, Xuxu Sun ${ }^{1}$, Ibrahim Nassour ${ }^{1,4}$, Jiyoung Park ${ }^{5}$, Alexandra Ghaben ${ }^{5}$, Tao Wang ${ }^{2}$, Sam C. Wang ${ }^{1,4}$, Philipp E. Scherer ${ }^{5}$, Cigall Kadoch $^{3}$ and Hao Zhu ${ }^{1,6, *}$

\section{Affiliations:}

${ }^{1}$ Children's Research Institute, Departments of Pediatrics and Internal Medicine, Center for Regenerative Science and Medicine, University of Texas Southwestern Medical Center, Dallas, TX 75390, USA.

${ }^{2}$ Quantitative Biomedical Research Center, Department of Population and Data Sciences, University of Texas Southwestern Medical Center, Dallas, TX 75390, USA

${ }^{3}$ Dana-Farber Cancer Institute and Harvard Medical School, Boston, MA, USA 02215. Broad Institute of MIT and Harvard, Cambridge, MA, USA

${ }^{4}$ Department of Surgery, University of Texas Southwestern Medical Center, Dallas, TX 75390, USA.

${ }^{5}$ Touchstone Diabetes Center, Department of Internal Medicine, the University of Texas Southwestern Medical Center, Dallas, TX, USA.

${ }^{6}$ Lead author

${ }^{*}$ Correspondence to:

Hao Zhu

Email: Hao.Zhu@utsouthwestern.edu

Phone: (214) 648-2850 


\section{Summary}

The dynamic regulation of $\beta$-cell abundance is poorly understood. Since chromatin remodeling plays critical roles in liver regeneration, these mechanisms could be generally important for regeneration in other tissues. Here we show that the ARID1A mammalian SWI/SNF complex subunit is a critical regulator of $\beta$-cell regeneration. Arid1a is highly expressed in quiescent $\beta$-cells but is physiologically suppressed when $\beta$-cells proliferate during pregnancy or after pancreas resection. Whole-body Arid1a knockout mice were protected against streptozotocin induced diabetes. Cell-type and temporally specific genetic dissection showed that $\beta$-cell specific Arid1a deletion could potentiate $\beta$-cell regeneration in multiple contexts. Transcriptomic and epigenomic profiling of mutant islets revealed increased Neuregulin-ERBB-NR4A signaling. Functionally, ERBB3 overexpression in $\beta$-cells was sufficient to protect against diabetes, and chemical inhibition of ERBB or NR4A was able to block increased regeneration associated with Arid1a loss. mSWI/SNF complex activity is a barrier to $\beta$-cell regeneration in physiologic and disease states. 


\section{Introduction}

Understanding how to recover endogenous $\beta$-cells in disease might ultimately depend on understanding how $\beta$-cell abundance is controlled in the first place. The molecular circuitry that dictates total $\beta$-cell mass during development and dynamic physiologic situations such as pregnancy is poorly understood. It is also unclear if the same mechanisms regulate $\beta$-cell expansion after injuries and during disease processes. One unexploited therapeutic strategy is to expand endogenous $\beta$-cells in type 1 and 2 diabetes, settings in which normal regenerative capacity cannot fully compensate for $\beta$-cell loss. Given the importance of dedifferentiation and proliferation in profoundly regenerative organisms such as zebrafish and planaria (Jopling et al., 2011), it stands to reason that facilitating chromatin state changes that occur physiologically could also increase self-renewal capacity in mammalian tissues. We hypothesized that the mammalian SWI/SNF (mSWI/SNF) ATP-dependent chromatin remodeling complex, known to promote terminal differentiation (Han et al., 2019; Hota et al., 2019; Sun et al., 2016b; Yu et al., 2013; Zhang et al., 2019, 2016) and also known to limit regeneration after liver injuries (Sun et al., 2016b), might operate as a general repressor of regeneration in tissues other than the liver. Given key similarities between $\beta$-cell and hepatocyte self-renewal, we reasoned that $\mathrm{mSWI} / \mathrm{SNF}$ might also suppress regenerative capacity in $\beta$-cells, a centrally important cell type in metabolic disease.

mSWI/SNF are large 10-15 component complexes containing a core ATPase (BRG1 or BRM, also known as SMARCA4 and SMARCA2), plus non-catalytic subunits that influence targeting and complex activities (Wu et al., 2009) such as the mutually exclusive ARID1A (BAF250A) and ARID1B (BAF250B) subunits, which together define the canonical BAF family of mSWI/SNF complexes. The loss of ARID1A alters the assembly of the ATPase module (Mashtalir et al., 2018), influencing canonical BAF complex targeting and DNA accessibility, resulting in global changes in gene regulation (Chandler et al., 2013; Kelso et al., 2017; Mathur et al., 2017; Nakayama et al., 2017; Pan et al., 2019; Sun et al., 2018). How the molecular consequences of $\mathrm{mSWI} / \mathrm{SNF}$ perturbation relate to physiologic phenotypes and disease outcomes is an active area of exploration.

Intriguingly, ARID1A and other mSWI/SNF components are downregulated during islet expansion, findings shared with other regenerative tissues such as the liver (Sun et al., 2016b). We demonstrated that this downregulation is functionally important by using spatially and 
temporally specific conditional knockout mouse models subjected to multiple types of $\beta$-cell injuries. These findings support the idea that physiological events that occur during regeneration can be further amplified to accelerate tissue healing. Molecular dissection of the events occurring downstream of ARID1A loss showed that NRG-ERBB-NR4A signaling activities were increased. Interestingly, the ERBB protein family (EGFR/ERBB1, ERBB2, ERBB3, and ERBB4) of transmembrane receptor tyrosine kinases (RTKs) have been implicated in diabetes (Miettinen et al., 2006; Oh et al., 2011; Song et al., 2016). Remarkably, SNPs in the human ERBB3 locus are among the strongest signals in T1D GWAS but how these SNPs affect ERBB signalling and $\beta$-cell biology were previously unknown (Bradfield et al., 2011; Nikitin et al., 2010; Sun et al., 2016a). Here, we show that ARID1A-containing SWI/SNF complexes have a major and unexpected role in $\beta$-cell regeneration, operating in large part through pathways that were previously implicated by GWAS studies in diabetogenesis. This expands our emerging understanding of $\mathrm{mSWI}$ SNF as a central regulator of tissue regeneration, and underscores the need to understand chromatin remodeling mechanisms that may represent therapeutic targets in regeneration.

\section{Results}

\section{Arid1a expression is suppressed during physiologic $\beta$-cell expansion}

ARID1A-containing SWI/SNF chromatin remodeling complexes (or canonical BAF complexes) drive terminal differentiation and block proliferation by regulating chromatin accessibility at loci targeted by lineage specific transcription factors (Alver et al., 2017; Spaeth et al., 2019; Vierbuchen et al., 2017). In the absence of ARID1A, increased liver regeneration and accelerated wound healing were observed (Sun et al., 2016b). Although the primary mechanism for the homeostatic maintenance of adult $\beta$-cells is self-duplication, majority of adult pancreatic $\beta$-cells are still mostly in post-mitotic state (Dor et al., 2004; Meier et al., 2008). We wondered if the inhibition of SWI/SNF activity could increase their proliferative potential. Genes that encode components of the SWI/SNF complex are expressed at high levels in the mouse $\beta$-cell line MIN6 and primary pancreatic islets compared to the mouse a-cell line ATC1 and primary hepatocytes (Figure 1A). In particular, Arid1a is highly expressed in quiescent $\beta$-cells within the islet (Figure 1B). Next, we examined Arid1a expression in islets under conditions that demand $\beta$-cell expansion, such as pregnancy and $50 \%$ partial pancreatectomy (PPx) (Ackermann 
Misfeldt et al., 2008; Rieck and Kaestner, 2010). Arid1a mRNA was lower in maternal islets during pregnancy-associated $\beta$-cell expansion, and protein levels declined until the time of birth (Figure 1C,D). Similarly, ARID1A protein levels were reduced during regeneration induced by PPx (Figure 1E).

We asked if signals known to elicit $\beta$-cell hyperplasia can influence the levels of Arid1a mRNA. We isolated wild-type (WT) mouse islets and treated with glucagon-like peptide-1 (GLP-1), high glucose, insulin-like growth factor-2 (IGF2), and interleukin-1 $\beta$ (IL-1 $\beta$ ), all factors known to drive $\beta$-cell proliferation (Alismail and Jin, 2014; De León et al., 2003; Gaddy et al., 2010; Hajmrle et al., 2016; Porat et al., 2011). Each factor suppressed Arid1a mRNA levels in islets, providing a potential molecular rationale for how the suppression of Arid1a during $\beta$-cell expansion is linked to upstream signals (Figure 1F). These results indicated dynamic regulation of ARID1A and SWI/SNF complex components during $\beta$-cell expansion and regeneration, suggesting functional roles during these processes.

\section{Whole-body Arid1a deletion protected against type I diabetes}

To determine if ARID1A restrains $\beta$-cell mass in adults, we inducibly deleted Arid1a in a whole body "Arid1a KO" mouse strain. Global deletion was induced with tamoxifen in adult Ubiquitin-CreER; Arid1a ${ }^{F / F I}$ (Ubc-CreER; Arid1a ${ }^{F / F I}$ ) mice (Figure 2A). Almost complete absence of the flox band and protein levels were observed (Figure 2B-D). Whole body Arid1a KO mice did not show overt signs of disease and appeared to be healthy. At baseline, whole body glucose tolerance was unchanged, indicating that ARID1A is not required for the regulation of glucose homeostasis or uptake under non-injury, basal conditions (Figure 2E). To determine if Arid1a KO mice were more able to cope with $\beta$-cell destruction in a type 1 diabetes (T1D) model, we subjected mice to streptozotocin (STZ), a chemical that ablates insulin-producing $\beta$-cells (Figure 2A). After STZ, Arid1a KO mice were almost completely protected against the development of diabetes as measured by fed state glucose levels (Figure 2F) and glucose tolerance testing (GTT) (Figure 2G). This left the possibility of either insulin secretion or insulin sensitivity changes, particularly because Arid1a was deleted in all tissues. Mice subjected to STZ did not show changes in insulin sensitivity based on insulin tolerance testing (ITT), suggesting no substantial metabolic impact of Arid1a loss on peripheral tissues, despite efficient deletion in those tissues (Figure 2H). However, Arid1a KO mice produced higher levels of 
insulin after glucose administration (Figure 2I), suggesting a $\beta$-cell mediated mechanism of protection against or response to STZ-induced T1D.

\section{Arid1a deficiency leads to a $\beta$-cell-mediated anti-diabetic phenotype}

Given that the whole body KO mice have lost Arid1a in multiple cell types, it was possible that non- $\beta$-cell autonomous mechanisms might have been at play. In WT mice, ARID1A is expressed in most acinar, all duct, and all islet cells. To rule out potential paracrine or endocrine effects, we used Ptf1a-Cre; Arid1a ${ }^{F / F I}$ mice to induce deletion in non- $\beta$-cells in the pancreas. Ptf1a is a transcription factor that is expressed in all cell types in the pancreas starting around E9.5 (Kawaguchi et al., 2002). In Ptf1a-Cre; Arid1a ${ }^{F I / F I}$ mice, ARID1A was lost in all acinar and most duct cells but ARID1A protein was retained in the islets, as shown recently (Wang et al., 2019a, 2019b). GTT did not reveal any differences in glucose clearance efficiency between WT, Ptf1a-Cre; Arid1a ${ }^{+/ F I}$, or Ptf1a-Cre; Arid1a ${ }^{F I / F I}$ mice (Figure S1A). Following STZ treatment (Figure S1B), there were also no differences in blood glucose measurements over the course of 1 month (Figure S1C), ruling out a potential contribution from acinar or duct cells to the phenotypes observed in whole-body KO mice.

Next, we generated a temporally regulated, $\beta$-cell specific model of Arid1a loss. This model allowed us to induce $\beta$-cell specific deletion of Arid1a after $\beta$-cell injuries, thus giving us the ability to answer questions specifically about $\beta$-cell regeneration, rather than cell survival in the presence of injuries. We employed a MIP (Mouse Insulin Promoter)-rtTA; TRE (Tetracycline Responsive Element promoter)-Cre transgenic system to allow such genetic control (Figure 3A) (Kusminski et al., 2016). MIP-rtTA; TRE-Cre; Arid1a ${ }^{\text {FIFI }}$ (Arid1a $\left.\beta K O\right)$ mice and littermate controls without Cre and/or rtTA were used to model cell-type and temporally specific Arid1a deletion. Notably, some Arid1a floxed band was still present in islets, potentially due to $\alpha, \delta$ and Y cell retention of the Arid1a floxed allele (Figure 3B). Despite the absence of ARID1A nuclear staining (Figure 3C), islet cell morphology was normal. Two weeks post-deletion, the ability to clear glucose after GTT challenge was unaltered (Figure 3D), suggesting that there was no change in $\beta$-cell function at baseline. Next, both groups of mice were given five doses of STZ $(50 \mathrm{mg} / \mathrm{kg}$ per day $x 5$ days), then fed doxycycline $(1 \mathrm{mg} / \mathrm{mL})$ water to conditionally induce Arid1a deletion in the $\beta$-cells of the experimental group. $\beta$-cell specific deletion of Arid1a after STZ administration prevented the rise of blood glucose to pathological levels (Figure 3E), and reduced the profound weight loss associated with T1D (Figure 3F). In the setting of STZ, Arid1a 
ßKO showed higher insulin levels than control mice. Not surprisingly, insulin levels were still lower in the STZ treated Arid1a ßKO group than uninjured WT mice since STZ was still able to ablate a subset of $\beta$-cells (Figure 3G). These data showed that Arid1a deficiency in $\beta$-cells was sufficient to preserve insulin production in response to STZ-induced T1D.

\section{Arid1a loss results in increased $\beta$-cell survival and proliferation}

We examined islets before and after STZ injury. Insulin staining in WT and Arid1a $\beta$ KO islets at baseline showed no differences. Thirty days after STZ, Arid1a $\beta$ KO mice had more $\beta$-cells than did WT mice (Figure 4A). In addition, WT mice showed considerable a-cell expansion as marked by glucagon staining (Figure 4B). Because resistance to diabetes could have been mediated by either a failure to lose $\beta$-cells or an increased ability to regenerate $\beta$-cells, we examined proliferation in the insulin expressing $\beta$-cell compartment. Arid1a deficient $\beta$-cells had greater numbers of Ki-67 positive cells. In the WT setting, a majority of the proliferating cells were positioned in the islet periphery, which is the non-insulin expressing compartment (Figure 4C,D). We then wondered whether the observed increase in proliferation after STZ had any effect on islet number or area. Although we did not detect any increase in the number of islets per unit of pancreas area (Figure 4E), there was a trend towards increased individual islet area $(p=0.06)$ (Figure 4F) in $\beta K O$ mice 3 weeks after STZ.

Given the remote possibility that Arid1a deficient $\beta$-cells could be protected from T1D due to a lack of STZ induced destruction rather than increased regeneration, we also performed PPx, a surgical assay that does not rely on the ability of cells to metabolize chemical toxins such as STZ. PPx leads to $\beta$-cell proliferation that compensates for overall islet loss. Six days after resection, the islets from regenerated pancreata of $\beta K O$ mice showed a significant increase in $\beta$-cell proliferation as measured by $\mathrm{Ki}-67$ positive cell number within the insulin expressing compartment (Figure 4G). In summary, Arid1a loss in $\beta$-cells did not constitutively enforce islet overgrowth in the absence of injury but increased proliferation after chemical and surgical injuries.

\section{Phenotypes associated with Arid1a loss are dependent on EGF/Neuregulin hyperactivation}

To probe the transcriptional programs associated with Arid1a deletion, we performed RNA-seq on islets from control and KO mice, before and after pancreatectomy. Of 2796 
differentially-expressed genes, 1586 were up and 1210 were downregulated (Figure 5A). Gene Set Enrichment Analysis (GSEA) performed on differentially expressed genes showed that genes involved in the neuregulin (NRG) and epidermal growth factor (EGF) pathways were collectively overproduced in KO islets (Figure 5B \& Figure 5A right inset). EGF and NRG are the ligands that activate ERBB family of RTKs. Binding of the EGF family growth factors leads to dimerization and phosphorylation of RTKs which leads to the activation of ERK1/2 and phosphatidylinositol 3-kinase (PI3K) signaling pathways (Yarden and Sliwkowski, 2001).

To functionally interrogate the transcriptomic findings, we performed shRNA knockdown of Arid1a in BTC6 and MIN6 immortalized $\beta$-cell lines to determine if Arid1a loss might cause increased dependency on NRG/EGF signaling. Arid1a knockdown in BTC6 and MIN6 caused increased phospho-EGFR ( $p$-EGFR) after EGF ligand exposure, indicating potentiation of the pathway (Figure 5C). In addition, EGFR/ERBB inhibition with the small molecule inhibitors erlotinib and canertinib were able to ablate p-EGFR in the presence of EGF (Figure 5D). Similar proliferative phenotypes were confirmed with Arid1a shRNA in MIN6 cells and these small molecule inhibitors selectively abrogated the hyperproliferation associated with Arid1a knockdown (Figure 5E).

To corroborate the shRNA results using another in vitro model, we generated Arid1a deficient MIN6 $\beta$-cells using CRISPR. Multiple single cell MIN6 clones for each genotype were expanded and confirmed for Arid1a loss (Figure 5F). Similar to $\beta$-cells from mouse islets, Arid1a KO MIN6 clones grew more rapidly than Gal4 targeted control clones (Figure 5G). Genes identified in the RNA-seq experiment were also upregulated in KO vs. control islets (Nr4a1, Nr4a2, Srf, JunB; see Figure $\mathbf{5 H}$ ). Collectively, these data show that Arid1a loss in $\beta$-cells causes a preferential dependency on ERBB signalling.

To determine if differentially expressed genes from the RNA-Seq analysis also showed changes in epigenetic marks, we performed H3K27 acetylation (H3K27ac) ChIP-seq. Sites marked by $\mathrm{H} 3 \mathrm{~K} 27 \mathrm{ac}$ were clustered into groups that increased or decreased in H3K27ac in response to Arid1a deletion (Figure 5I). Interestingly, many sites increased in H3K27ac abundance in the Arid1a heterozygous and homozygous clones (clusters 3 and 4). The clusters containing sites that changed in H3K27ac occupancy upon Arid1a loss were largely composed of sites at distal regions (Figure $\mathbf{5 J}$ ), which is consistent with the disruption of canonical BAF complexes (Kelso et al., 2017; Mathur et al., 2017; Vierbuchen et al., 2017). Sites that increased in H3K27ac upon 
Arid1a loss included Nrg4 and Egfr, consistent with transcriptional hyperactivation in the Arid1a-deficient setting (Figure 5K). GO biological processes associated with increased acetylation of H3K27 in the Arid1a knockout populations in clusters 3 and 4 contain terms involving glucocorticoid biosynthesis and hormone metabolism, and the associated mouse phenotypes included abnormal insulin secretion and pancreas secretion (Figure S2A and Figure S2B). These observations are consistent with differential regulation of pathways affecting glucose metabolism and insulin secretion in $\mathrm{KO}$ mouse $\beta$-cells.

To challenge the idea that Arid1a deletion causes more proliferation through the preferential promotion of ERBB signaling as opposed to a more generalized activation of multiple mitogenic signal pathways, we performed a small molecule screen to identify pathways that Arid1a KO cells are more dependent on for growth and survival. We screened 300 kinase inhibitors on previously generated immortalized $\mathrm{H} 2.35$ cells isogenic for Arid1a deletion (Figure S3A). Interestingly, ERBB family inhibitors were significantly enriched among treatments that induced the most prominent reductions in cell viability $(P$-value $=5.8 \mathrm{e}-6)$. There were 3 ERBB family inhibitors (Afatinib, WZ8040, canertinib) among the top 10 kinase inhibitors associated with the highest reduction of growth/viability in Arid1a KO cells (Figure S3B). Together with the islet transcriptome data, these observations established a strong functional connection between ARID1A and ERBB.

\section{$E R B B 3$ overexpression independently increases $\beta$-cell regeneration}

In order to perform gain-of-function studies for ERBB signalling, we first asked which $E R B B$ receptor loci ARID1A might be acting through. ChIP-seq data on human liver cell line HepG2 showed binding of ARID1A and core SWI/SNF component SNF5 on the promoters of ERBB2 and ERBB3 (Figure 6A) (Raab et al., 2015). In addition, multiple human GWAS studies have shown strong associations between a SNP (rs2292239) in ERBB3 and T1D (Barrett et al., 2009; Kaur et al., 2016; Sun et al., 2016a; Todd et al., 2007). Based on these observations, we reasoned that ARID1A could have a genetic interaction with ERBB3. Because it is still unknown whether or not an increase or decrease of ERBB3 in $\beta$-cells or other tissues is protective in human T1D, we generated a $\beta$-cell specific human ERBB3 overexpression mouse model (MIP-rtTA; TRE-ERBB3) (Figure 6B). Strikingly, inducible overexpression of ERBB3 alone was sufficient to mediate resistance to STZ-induced T1D (Figure 6C). This is the first functional confirmation of an effect for ERBB3 on a T1D model. 
To determine if there is an epistatic relationship between ERBB3 and Arid1a, we genetically manipulated both genes in vivo. We hypothesized that if the two genes act via distinct pathways, ERBB3 overexpression in an Arid1a deficient background would have a synergistic effect (Figure 6D), whereas if both genes are within the same pathway, then there would be little to no additive effect. While mice with either $\beta$-cell specific ERBB3 overexpression or Arid1a loss showed protection against STZ-induced diabetes, simultaneous perturbations did not synergize to increase the protective effect (Figure 6E). The lack of a synergistic effect suggests that either these genes are acting in the same pathway, or that the effects are individually saturated. These results for the first time validate the human GWAS signals for ERBB3 in an animal model and support the hypothesis that Arid1a loss is operating through increases in ERBB signaling.

\section{Pan-ERBB and NR4A1 inhibition ablated the Arid1a KO phenotype in vivo}

We sought to determine whether pro-proliferative effects of Arid1a loss were mediated through ERBB activation in vivo as well as in vitro. We first tested the in vivo relevance of these results by examining whole body Ubc-CreER; Arid1a WT and KO mice described earlier. After STZ mediated islet ablation, mice were given daily doses of canertinib, an intervention that abolished the anti-diabetic effects of Arid1a loss (Figure 7A, compare to Figure 2F which did not include canertinib). We reasoned that ERBB inhibition in the STZ model could have been influenced by cell death in addition to proliferation, so we also performed the PPx assay to assess $\beta$-cell proliferation after surgical injury. Here, $\beta$-cell specific Arid1a deletion was induced one week before PPx and drug treatments were started one day before and continued until 6 days after PPx, which is the apex for $\beta$-cell proliferation (Peshavaria et al., 2006). The increase in $\mathrm{Ki}-67 /$ insulin double positive $\beta$-cells became more pronounced in the regenerating pancreas (Figure 7B, top row \& 7C). Again, canertinib abolished the pro-proliferative effect seen in KO islets (Figure 7B, middle row \& 7C). These results showed that ERBB signaling in part mediates the $\beta$-cell regenerating effects of Arid1a deficiency in vivo.

Because ARID1A likely influences a large network of genes that regulate differentiation and regenerative capacity, we next asked if other genes downstream of ERBB signaling exert the effects of Arid1a loss. To further examine important functional networks resulting Arid1a KO phenotype, a protein-protein interaction network of overexpressed genes from the RNA-seq data was created using the STRING database (Szklarczyk et al., 2015). This interaction network 
showed that the SRF/JUN/FOS/EGR1 transcription factors and the NR4A family of nuclear receptor transcription factors were tightly connected and clustered together (Figure 7D). Indeed, these genes also came up under the GSEA datasets that were classified as "EGF/NRG1 signaling up" (Figure 5A) suggesting that they are downstream of ERBB signaling. The overexpression of these transcription factors were previously established as one of the hallmarks of immature, proliferative $\beta$-cells (Zeng et al., 2017). We validated higher protein levels of c-FOS and p-NR4A1 in KO islets (Figure 7E). We then asked if ARID1A relays its effects in part through NR4A1 in vivo by treating mice that had undergone PPx with an NR4A1 antagonist (C-DIM8), in a similar fashion as the canertinib experiments. C-DIM8 also abolished the increased proliferation normally seen in Arid1a KO $\beta$-cells (Figure 7B, bottom row \& 7C), suggesting that increased NR4A1 activity is additionally required for the phenotypic effects of Arid1a loss.

\section{Discussion}

Preserving or replenishing endogenous pancreatic $\beta$-cells is a major challenge in diabetes. To ultimately meet these goals, there is a need to understand the factors limiting $\beta$-cell proliferation. Although engineering strategies such as inducible pluripotent stem cell to $\beta$-cell conversion or the transdifferentiation of other cell types to $\beta$-cells are emerging (Aguayo-Mazzucato and Bonner-Weir, 2018), targeting endogenous regeneration of $\beta$-cells has potential advantages since this occurs in development, pregnancy, and obesity to meet increased physiological demands for insulin production. Here, we have identified Arid1a as a factor whose suppression is required for gestational $\beta$-cell expansion and is sufficient for $\beta$-cell replenishment after multiple pancreatic injuries. Previous studies have shown that BRG1 and BRM-containing SWI/SNF complexes have context-dependent roles in modulating PDX1 activity in $\beta$-cell development and function (McKenna et al., 2015; Spaeth et al., 2019). Wei et. al. reported that Vitamin $D$ ligand causes the Vitamin $D$ receptor to interact with an active BRD7/pBAF rather than an inactive BRD9/ncBAF complex, which reduces $\beta$-cell failure and slows diabetes progression (Wei et al., 2018). Our study is the first to highlight Arid1a and SWI/SNF as an important epigenetic node that regulates the regenerative capacity of $\beta$-cells.

By functionally validating EGF/NRG1 signaling as a critical effector of ARID1A suppression, we connected two previously unrelated, but prominent regeneration networks. EGF signaling is known to be important for liver, heart, and pancreas regeneration (D'Uva et al., 2015; 
Gemberling et al., 2015; Song et al., 2016; Sun et al., 2016b). GWAS studies implicated ERBB3 as one of the most significant T1D gene loci, but it is not clear if ERBB3 expression is positively or negatively influencing T1D risk, and if ERBB3 is acting in $\beta$-cells, immune cells, or peripheral tissues (Barrett et al., 2009; Kaur et al., 2016; Sun et al., 2016a; Todd et al., 2007). For the first time, we determined that ERBB3 overexpression in $\beta$-cells is sufficient to protect against diabetes. The fact that overexpression did not add to the protective phenotype of Arid1a deletion is consistent with the idea that ARID1A loss acts through ERBB signaling.

Mechanistically, our results implicate a network of EGF/NRG1 signaling genes. These include $\mathrm{Nr4a}$ nuclear receptor genes and a set of nutrient-responsive immediate early genes (JunB, Fos, Egr1) and their upstream activator Srf (Mina et al., 2015). Interestingly, EGR binding sites represented the most dynamic chromatin regions during whole body regeneration of Hofstenia miamia, an acoel worm that is capable of whole body regeneration. In these worms, EGR is essential for regeneration through its activities as a pioneer factor that directly regulated wound-induced genes including the EGFR ligands nrg-1 and nrg-2 (Gehrke et al., 2019). In addition, Zeng et al. recently used single cell transcriptomics to categorize $\beta$-cells from less to most mature. They showed that Srf/JunB/Fos/Egr1 were among the most downregulated genes during postnatal $\beta$-cell maturation and comprise a signature of less mature, proliferative $\beta$-cells. Importantly, overexpression of upstream activator Srf in islets did not impair insulin secretion (Zeng et al., 2017). Our data suggest that ARID1A containing complexes may negatively regulate this larger network of pro-regeneration genes. Importantly, $\beta$-cells without ARID1A are poised for proliferation while still able to maintain insulin production.

Our study highlights targets that could be investigated for their therapeutic potential in future studies. In contrast to the majority of nuclear receptors, the NR4A family of nuclear receptors do not have an identified physiological ligand, therefore their activity is mainly regulated at the level of protein expression and post-translational modifications (Safe et al., 2016). However, there is a agonist, Cytosporone-B, that can selectively increase the transcriptional activity of Nr4a1 (Zhan et al., 2008). Whether this agonist could be utilized to improve the regenerative potential of $\beta$-cells is an area of interest. A more direct way to tackle this problem would be to use specific inhibitors targeting ARID1A or BAF complexes. There is a major interest in the field to develop small molecules to manipulate activities of SWI/SNF complex, and if successful, it will be interesting to determine if such inhibitors might be effective in diabetes. 


\section{Acknowledgements}

We would like to thank Michael Kalwat and Melanie H. Cobb for sharing MIN6 cell line. Histology Pathology Core (John Shelton), CRI Sequencing Core (Jian Xu, Xin Liu, Yoon Jung Kim) and CRI Mouse Genome Engineering Core (Yu Zhang) contributed to this project. We would like to thank Eric Olson, Jiang $\mathrm{Wu}$, and Jian $\mathrm{Xu}$ for their valuable feedback during this project. C.C. and X.S. were supported by The Hamon Center for Regenerative Science and Medicine (CRSM) Trainee Fellowships. T.W. is supported by a R03ES026397-01 and CPRIT (RP150596). H.Z. was supported by the Pollack Foundation, an NIH/NIDDK R01 grant (DK111588), a Burroughs Wellcome Career Award for Medical Scientists, a CPRIT Scholar Award (R1209).

\section{Author Contributions}

C.C. and J.C. designed and performed the experiments and wrote the paper. S.S. maintained the mouse colony and performed surgical procedures, islet isolations and data analysis. J.E.O and C.K.C performed H3K27Ac ChIP-Seq experiments. X.L. and Y.W. performed and T.W. supervised the bioinformatics analysis. L.L. generated MIN6 single cell clones and performed experiments. Z.W., X.S., I.N., J.P., A.G., P.E.S, S.C.W generated and analyzed mouse models. S.C.W. and C.K. edited the manuscript. H.Z. conceived and supervised the project and wrote the manuscript.

\section{Declaration of Interests}

The authors declare no competing interests.

\section{STAR Methods}

\section{LEAD CONTACT AND MATERIALS AVAILABILITY}

Further information and material requests should be directed to Dr. Hao Zhu (Hao.Zhu@utsouthwestern.edu).

\section{EXPERIMENTAL MODEL AND SUBJECT DETAILS}

Mice. All experiments on mice were approved by and handled in accordance with the guidelines of the Institutional Animal Care and Use Committee at UTSW. All experiments were performed 
in in age and gender controlled fashion. Male mice were used for STZ experiments. The transgenic mouse lines used are as follows and described before: Arid1a floxed mice (JAX stock \#027717) (Gao et al., 2008), Ptf1a-Cre (Kawaguchi et al., 2002), MIP-rtTA (Kusminski et al., 2016), TRE-Cre (JAX 006234) (Perl et al., 2002). TRE-HER3 mouse line was generated by Jiyoung Park and Alexandra Ghaben in Dr. Scherer's group and a paper with the detailed characterization of this mouse model is in preparation.

Cell Lines. The H2.35 (ATCC ${ }^{\circledR}$ CRL-1995 ${ }^{\mathrm{TM}}$ ) and BTC6 cells were obtained from ATCC

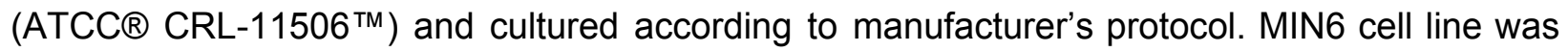
obtained from Dr. Melanie Cobb's lab and cultured in DMEM with 15\% Heat Inactivated Fetal Bovine Serum, 1\% L-Glutamine, 1\% Pen/Strep , 0.0005\% beta mercaptoethanol.

Mouse Islet Culture. Islets from adult mice were isolated and recovered overnight in culture medium (RPMI1640 with 10\% heat-inactivated FBS, L-glutamine and Pen/Strep) in the incubator and used for experiments.

\section{METHOD DETAILS}

Mouse pancreatic islet isolation and dispersion of islets. Islet isolation was done as described previously (Zmuda et al., 2011) with minor modifications. Briefly, Liberase TL (Roche, 05401020001) solution was prepared by dissolving $5 \mathrm{mg}$ lyophilized Liberase TL powder in $1 \mathrm{ml}$ sterile water so that the concentration is $5 \mathrm{mg} / \mathrm{ml}$ corresponding to 26 Wunsch units. Prior to use, this $1 \mathrm{ml}$ Liberase solution was added to $21.6 \mathrm{ml}$ serum free RPMI to obtain the working solution. Adult mice were perfused with $3 \mathrm{ml}$ Liberase TL working solution through the common bile duct cannulation and inflated pancreas was put into $2 \mathrm{ml}$ Liberase TL solution sitting on ice in $50 \mathrm{ml}$ falcon tube and incubated at $37^{\circ} \mathrm{C}$ water bath for $10-16$ minutes by shaking and reaction was stopped with the addition of RPMI containing serum. Islets were dissociated from the exocrine tissues by shaking vigorously several times followed by Histopaque 1077 (Sigma-Aldrich, 10771) density centrifugation (900g, 20 minutes, acceleration:2, deceleration: $0)$. Purified islets were collected from the interface and washed. Intact islets were hand-picked under the dissection microscope.

Islet mitogen experiments. Islets from male CD1 mice were isolated and recovered overnight in culture medium (RPMI1640 with 10\% heat-inactivated FBS, L-glutamine and Pen/Strep) in the incubator prior to various treatments. After recovering, islets were treated with either vehicle 
(culture medium with $11.1 \mathrm{mM}$ glucose), high glucose (culture medium with $25 \mathrm{mM}$ glucose), or with the addition of IGF2 (200nM), GLP-1 (100nM), IL1 $\beta(1 \mathrm{ng} / \mathrm{ml})$ for 6 hours and RNA was harvested for qPCR analysis.

Kinase inhibitor screen. SelleckChem customized library (Z49127) was a collection of 304 kinases inhibitors. See the supplementary table for a full list of inhibitors with additional details about their targets. Isogenic WT and Arid1a KO H2.35 transformed mouse liver hepatocyte cells were used in this kinase screen and treated with kinase inhibitors. To determine if EGFR family inhibitors is among the treatment conditions that induced the highest decrease in cell viability, we performed a hypergeometric test to examine the enrichment of treatments involving these inhibitors. Briefly, we ranked all treatment conditions in descending order according to observed cell viability loss, then, we counted the number of EGFR family inhibitor treatments among the top $10 \%$ conditions. The possibility of observing at least N EGFR family inhibitor treatments by chance is calculated and reported as the p-value of EGFR enrichment.

Generation of CAS9 single cell clones. Mouse Arid1a gRNA (GCTGCTGCTGATACGAAGGTTGG) was cloned into LentiGuide-puro plasmid (Addgene \#53963). LentiCas9-Blast plasmid was purchased from Addgene (Addgene \#53962). Active lentivirus was prepared in $293 \mathrm{~T}$ cells in $10-\mathrm{cm}$ dish. The day after seeding cells, each dish was transfected with pVSV-G, pLenti-gag pol, LentiCas9-puro-sGArid1a or LentiCas9-Blast plasmid by Lipofectamine 3000 (Life Technologies \# L3000015). Virus containing medium was collected at $60 \mathrm{~h}$ after transfection. For creating the Cas9 stable expressing cell line, cells were infected with Cas 9 lentivirus, followed by blasticidin selection at $2 \mathrm{ug} / \mathrm{ml}$ for 4 days. Then Cas 9 expressing cells were infected with Arid1a gRNA lentivirus. Three days after the infection, we selected cells for 3 days with puromycin at $2 \mathrm{ug} / \mathrm{ml}$. Then, 50 to 200 cells were plated in $15 \mathrm{~cm}$ dishes for single clone selection. Single clones were picked when they grew big enough and verified the genotype and Arid1a expression by PCR and Western blot.

In vivo drugs. Dox water $(1 \mathrm{~g} / \mathrm{L})$ was used to induce conditional deletion. Tamoxifen was dissolved (Sigma-Aldrich, T5648) in corn oil at a concentration of $20 \mathrm{mg} / \mathrm{ml}$ by sonication. 500uL of $20 \mathrm{mg} / \mathrm{mL}$ Tamoxifen by oral gavage for two consecutive days. $20 \mathrm{mg} / \mathrm{kg}$ canertinib (LC Laboratories NC0704940) was administered daily through oral gavage for one month in STZ experiments. Either 20 mg/kg canertinib or 20 mg/kg NR4A1 antagonist C-DIM8-DIM-C-pPhOH 
(Axon Medchem \# Axon 2827) was administered daily through gavage starting a day before PPx until sacrificing mice day 7 post-PPx.

Streptozotocin (STZ) injury. Mice were fasted for 4-6 hours prior to STZ injection. STZ (Sigma-Aldrich S0130) was dissolved in sodium-citrate (Sigma-Aldrich S4641) solution to a final concentration of $10 \mathrm{mg} / \mathrm{ml}$ freshly $10-15 \mathrm{~min}$ before the injection. Sodium-citrate solution was prepared by dissolving 1.47 gram of sodium-citrate powder in $50 \mathrm{ml} \mathrm{ddH2O}$, and adjusting the $\mathrm{pH}$ to 4.5. Prepared STZ solution was injected via intraperitoneal injection. Different dosage was used for different strain backgrounds as indicated in the figure legends since response to STZ is strain dependent.

Glucose tolerance test (GTT). After 16 hours fast, blood glucose was measured using a glucometer from tail tip blood before and multiple times within 2 hours of intraperitoneal injection of $2 \mathrm{~g} / \mathrm{kg} \mathrm{D}-(+)-G l u c o s e ~(S i g m a ~ A l d r i c h ~ \# ~ G 7528)$.

Insulin tolerance test (ITT). After $6 \mathrm{~h}$ fast, blood glucose was measured before intraperitoneal injection of insulin $(0.75 \mathrm{mU} / \mathrm{g}$ body $\mathrm{wt})$ and then 30, 60, 90 and $120 \mathrm{~min}$ after injection.

Partial pancreatectomy (PPx). PPx was performed as described (Martín et al., 1999) except that mice were not fasted overnight.

RNA Extraction and RT-qPCR. Total RNA from isolated mouse islets were extracted using TRIzol reagent (Invitrogen) or The RNeasy Plus Micro Kit (cat. no. 74034). For RT-qPCR of mRNAs, cDNA synthesis was performed with $1 \mathrm{mg}$ of total RNA using miScript II Reverse Transcription Kit (QIAGEN). See Supplemental Information for primers used in these experiments. Expression was measured using the $\Delta \Delta \mathrm{Ct}$ method.

Western blot. Isolated islets were lysed in RIPA buffer (Sigma R0278) supplemented with protease and phosphatase inhibitors. Protein concentration was determined by Pierce ${ }^{\mathrm{TM}}$ BCA Protein Assay Kit (Thermo Fisher \#23225). Western blots were performed in the standard fashion. The following antibodies were used: ARID1A (Sigma-Aldrich Cat\# HPA005456, RRID:AB_1078205), ARID1A (Santa Cruz Biotechnology Cat\# sc-32761, RRID:AB_673396), p-NR4A1 (Cell Signaling Technology Cat\# 5095, RRID:AB_10695108), phospho-EGFR (Tyr1068) (Cell Signaling Technology Cat\# 3777, RRID:AB_2096270), phospho-EGFR (Y1173) (Cell Signaling Technology Cat\# 4407, RRID:AB_331795), phospho-AKT (Ser473) (Cell Signaling Technology Cat\# 4060, RRID:AB_2315049), phospho-p44/42 (p-ERK) (Cell Signaling 
Technology Cat\# 9101, RRID:AB_331646), p44/42 (ERK) (Cell Signaling Technology Cat\# 9107, RRID:AB_10695739), c-FOS (Santa Cruz Biotechnology Cat\# sc-166940, RRID:AB_10609634), ß-Actin (Cell Signaling Technology Cat\# 4970, RRID:AB_2223172), Vinculin (Cell Signaling Technology Cat\# 13901, RRID:AB_2728768), anti-rabbit lgG, HRP-linked antibody (Cell Signaling Technology Cat\# 7074, RRID:AB_2099233) and anti-mouse lgG, HRP-linked antibody (Cell Signaling Technology Cat\# 7076, RRID:AB_330924).

Histology, immunohistochemistry, and immunofluorescence. Tissue samples were fixed in 4\% paraformaldehyde (PFA) and embedded in paraffin. H\&E staining was performed by the UTSW Histology Core Facility. Primary antibodies used: anti-rabbit Arid1a (Sigma-Aldrich Cat\# HPA005456, RRID:AB_1078205, used for IHC), anti-rabbit Glucagon (Cell Signaling Technology Cat\# 2760, RRID:AB_659831, used for IHC), anti-rabbit Insulin (Abcam Cat\# ab108326, RRID:AB_10861152, used for IHC), anti-rabbit Ki-67 (Abcam Cat\# ab15580, RRID:AB_443209, used for IF), anti-guinea pig Insulin (Abcam Cat\# ab7842, RRID:AB_306130, used for IF). Secondary antibodies used: Alexa Fluor 488 goat anti-rabbit IgG (Thermo Fisher; 11008; RRID:AB_10563748) goat polyclonal antibody to anti-Guinea pig IgG-Alexa 568 (Abcam, ab175714). For IHC, detection was performed with the Elite ABC Kit and DAB Substrate (Vector Laboratories) followed by hematoxylin counterstaining (Sigma). VECTASHIELD® Antifade Mounting Media with DAPI counterstain (Vector Labs, $\mathrm{H}-1200$ ) was used. For islet number and area calculation, H\&E sections were imaged using The NanoZoomer 2.0-HT whole slide imaging. Number of islets were counted in each slide. Whole H\&E section area and area of each individual islet in that H\&E section were measured using NDP.view software. To determine the proliferation, slices of pancreas were costained with insulin, Ki-67 and DAPI and all the islets present in the section were imaged using the same parameters. Channels were merged and images were analyzed in Image $\mathrm{J}$.

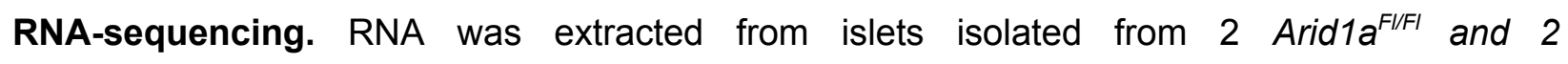
Ubiquitin-CreER; Arid1a ${ }^{F I F I}$ mice. RNA-seq libraries were prepared with the Ovation RNA-Seq Systems 1-16 (Nugen) and indexed libraries were multiplexed in a single flow cell and underwent 75 base pair single-end sequencing on an Illumina NextSeq500 using the High Output kit v2 (75 cycles) at the UTSW Children's Research Institute Sequencing Facility. 


\section{QUANTIFICATION AND STATISTICAL ANALYSIS}

RNA-Seq Analysis. RNA-Seq analysis was performed as described before (Celen et al., 2017). Briefly, adaptors and low quality sequences (Phred<20) were removed by trimming raw sequencing reads using galore package (http://www.bioinformatics.babraham.ac.uk/projects/trim galore/). The sequence reads were aligned to the GRCm38/mm10 with HiSAT2 (Kim et al., 2015). After duplicates removal by SAMtools ( $\mathrm{Li}$ et al., 2009), read counts were generated for the annotated genes based on GENCODE V20, using featureCounts (Harrow et al., 2012; Liao et al., 2014). Differential gene expression analysis was performed on edgeR, using FDR $<0.05$ as cutoff (McCarthy et al., 2012; Robinson et al., 2010). Heatmaps to visualize the data were generated by using GENE-E (Robinson et al., 2010).

GSEA. GSEA was used to determine significantly enriched gene sets. To perform GSEA analysis using RNA-seq data from WT and Arid1a KO islets, raw read counts from each sample were converted to $\mathrm{cpm}$ value (count per million) using the cpm function within edgeR $\mathrm{R}$ package. GSEA analysis was performed with a pre-ranked gene list by log fold change. GSEA was then performed against hallmark gene sets using default parameters (http://software.broadinstitute.org/gsea/index.jsp).

ChIP-seq Data Processing and Analysis. Sequencing of ChIP-seq samples was carried out using the Illumina NextSeq technology, and reads were demultiplexed with the bcl2fastq software tool. Read quality was evaluated by FASTQC, and read alignment to the hg19 genome was executed with Bowtie2 v2.29 in the -k 1 reporting mode (Langmead and Salzberg, 2012). Narrow peaks were detected using MACS2 v2.1.1 with a q-value cutoff of 0.001 and input as controls (Zhang et al., 2008). Output BAM files were transformed into BigWig track files using the "callpeak" function of MACS2 v2.1.1 with the "-B --SPMR" option followed by the use of the BEDTools (Quinlan and Hall, 2010) "sort" function and the UCSC utility "bedgraphToBigWig". BigWig track files were then input in IGV v2.4.3 for visualization. Heat maps were generated using ngsplot v2.61, which was also used to perform K-means clustering (Shen et al., 2014). Cis-regulatory function analysis was carried out using the GREAT online software suite (Argiras et al., 1987).

Statistical analysis. Variation is indicated using standard error presented as mean \pm SEM. Two-tailed Student's $t$-tests (two-sample equal variance) were used to test the significance of 
differences between the two groups. Statistical significance is displayed as * $(P<0.05)$, ** $(P<$ $0.01),{ }^{* *}(P<0.001),{ }^{* * * *}(P<0.0001)$. Statistical analyses were performed using GraphPad Prism unless otherwise indicated. Mice from multiple litters were used in the experiments. In STZ follow up experiments, mice were occasionally excluded from the analysis due to death.

\section{DATA AND CODE AVAILABILITY}

All sequencing data have been deposited in the GEO with the accession number (pending). 


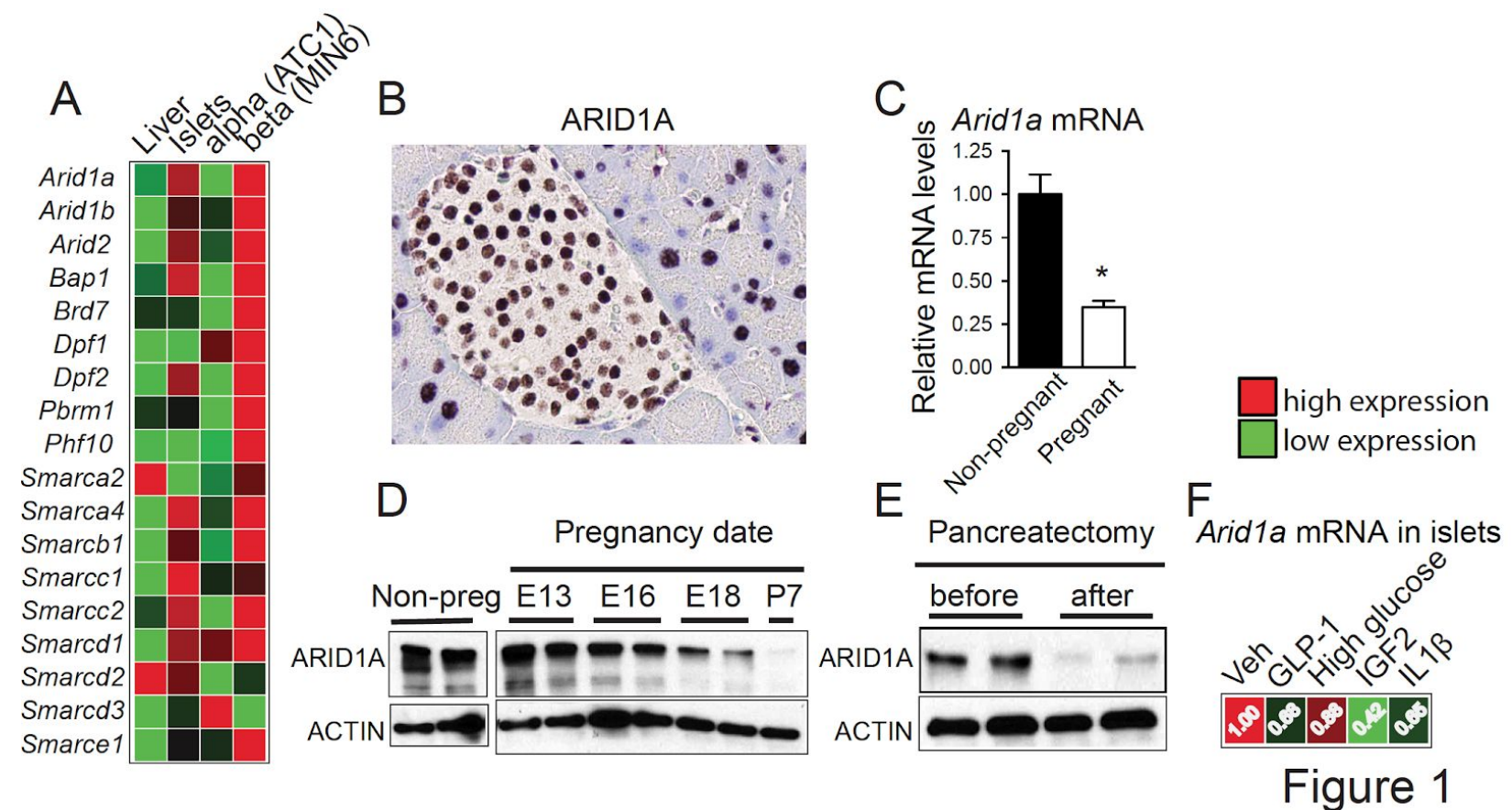

Figure 1. Arid1a expression is suppressed during physiologic $\beta$-cell expansion.

A. mRNA expression heat map of SWI/SNF components in mouse liver, mouse islet, alpha cell line (ATC1) and beta cell line (MIN6) using qPCR.

B. ARID1A immunostaining in the adult mouse pancreas. An islet is shown.

C. qPCR showing Arid1a mRNA levels in islets isolated from non-pregnant and pregnant females at gestational day 16 ( $n=3$ mice per group).

D. Western blot showing ARID1A levels in the islets of non-pregnant females and at different time points during gestation.

E. Western blot showing ARID1A levels in the islets before and 5 days after $50 \%$ pancreatectomy (PPX).

F. qPCR showing the reduction in Arid1a levels in isolated islets after 6 hours of treatment in culture with the following stimuli: GLP-1 (100 nM), high glucose (25 mM), IGF2 (200nM) and IL1 $\beta$ (1 ng/ml). 


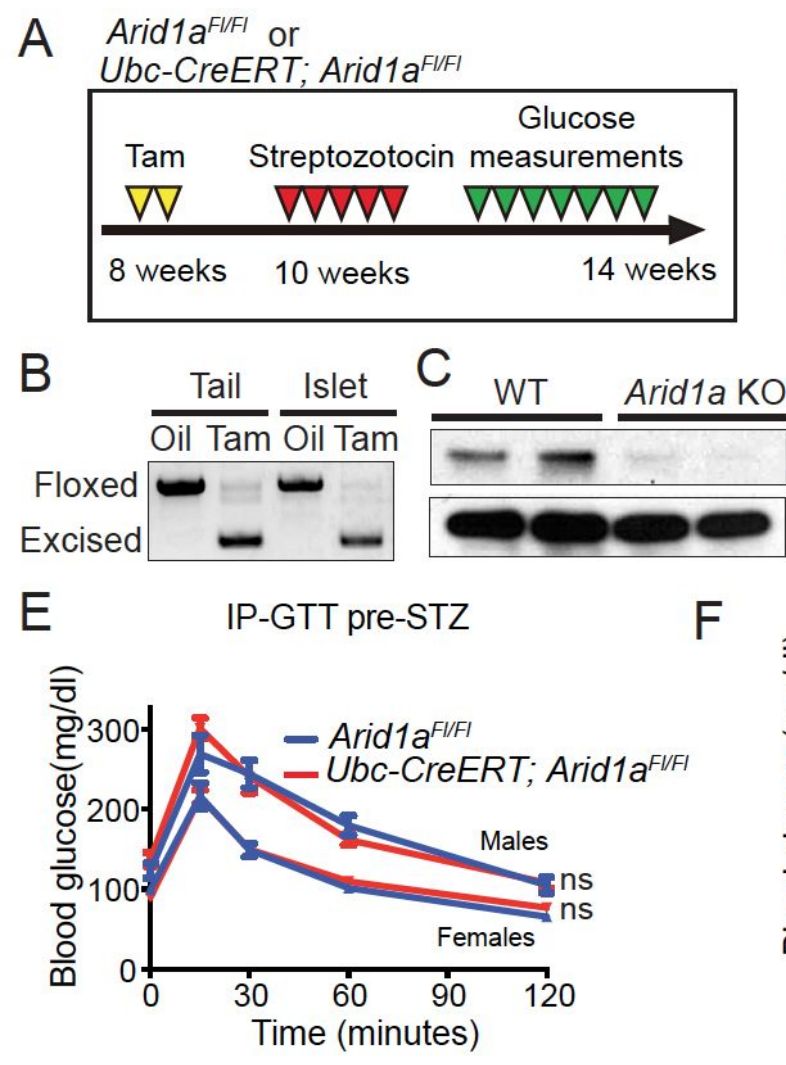

G

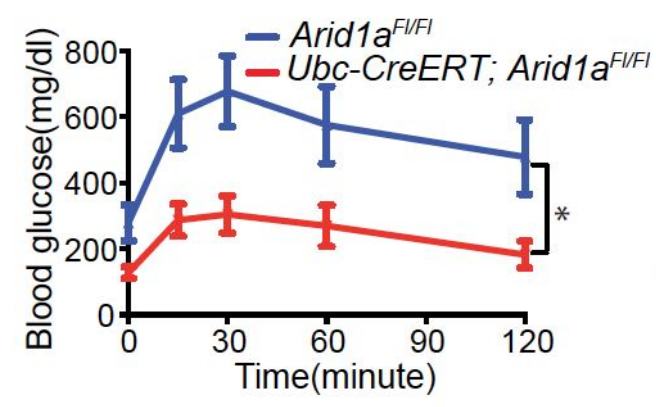

$\mathrm{H}$

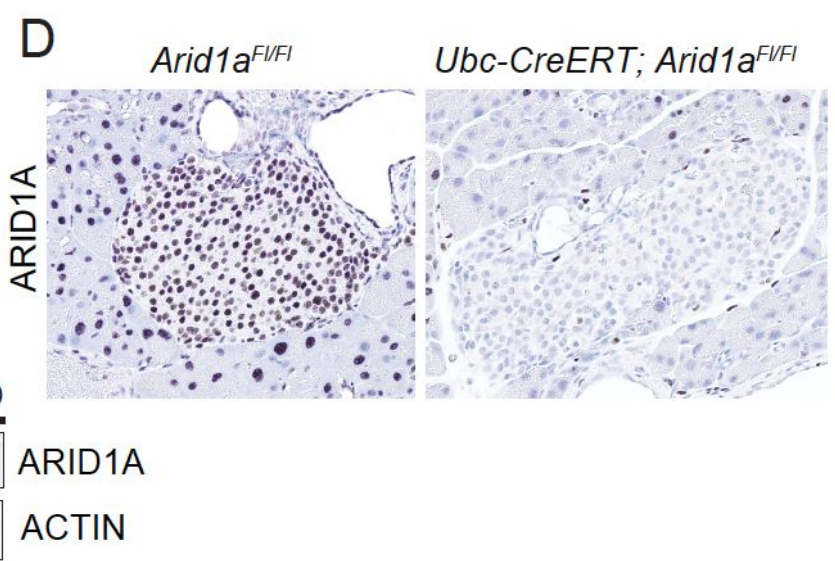

Fed state glucose post-STZ

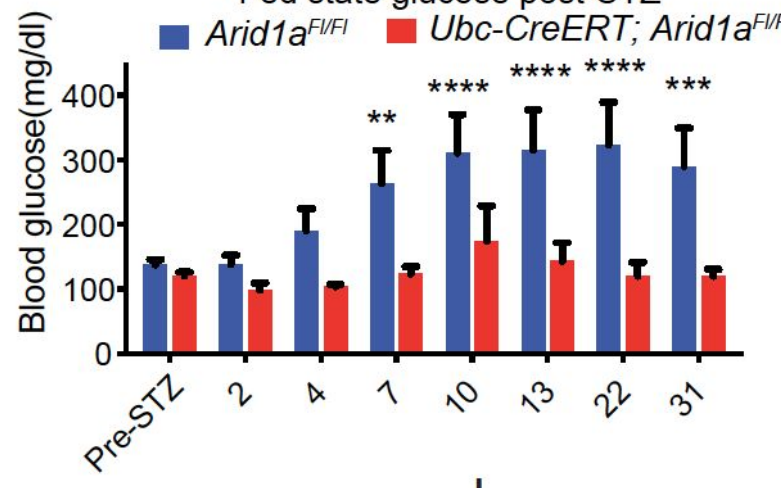

ITT post-STZ

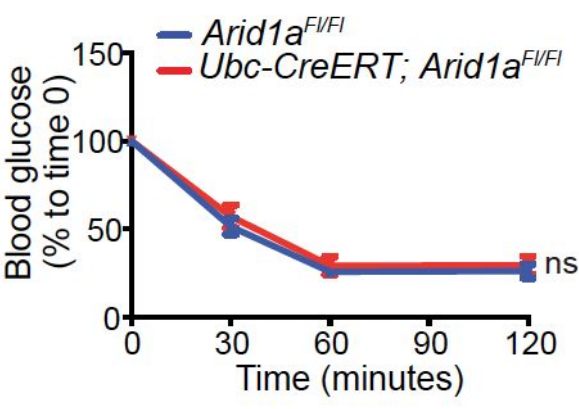

post-STZ

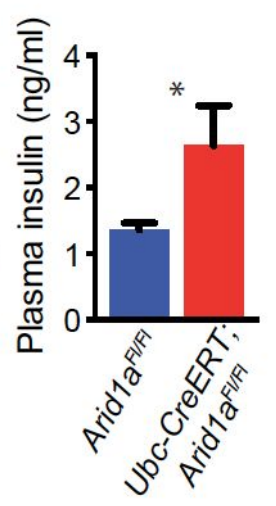

Figure 2 
Figure 2. Whole body Arid1a deletion protects against STZ-induced T1D.

A. Schema for STZ-induced diabetes studies in Arid1 $a^{\text {FI/FI }}$ control and Ubc-CreER; Arid1a $a^{\text {FIFI }}$ whole body $\mathrm{KO}$ mice. Two weeks after administration of $20 \mathrm{mg} / \mathrm{kg}$ tamoxifen oral gavage $x 2$ days, diabetes was induced by injecting $50 \mathrm{mg} / \mathrm{kg}$ STZ IP for 5 consecutive days.

B. Genotyping of tail and islets to assess the recombination at the Arid $1 a^{\text {FI/FI }}$ locus.

C. Western blot assessing the reduction in ARID1A protein levels.

D. Immunostaining of ARID1A in the adult pancreas of Ubc-CreER; Arid1a ${ }^{F / F I}$ mice.

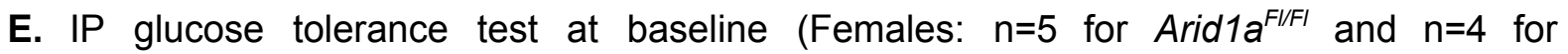

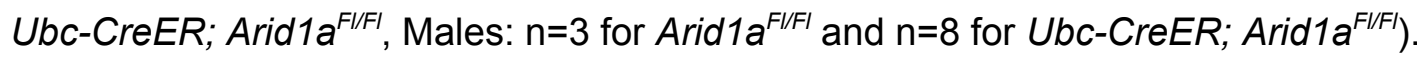

F. After STZ, fed state blood glucose was measured following $S T Z$ ( $n=11$ for Arid $1 a^{F I / F I}$ and $\mathrm{n}=9$ for Ubc-CreER; Arid1a ${ }^{\text {FI/FI }}$ ).

G. IP glucose tolerance test performed 2 weeks post-STZ.

H. Insulin tolerance test performed 2 weeks post-STZ.

I. Plasma insulin levels measured by ELISA 2 weeks post-STZ. 
A Inducible beta-cell specific Arid1a KO (Arid1a ßKO)

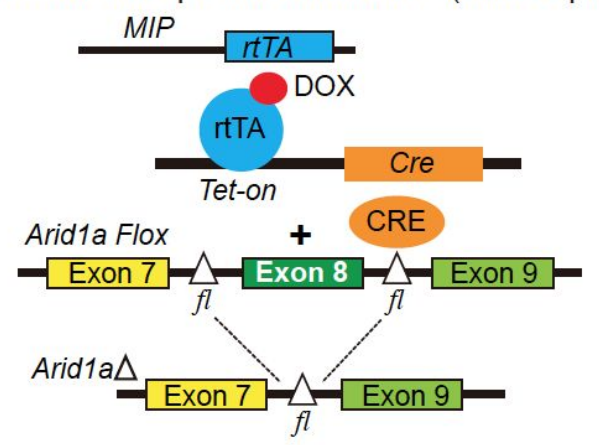

C

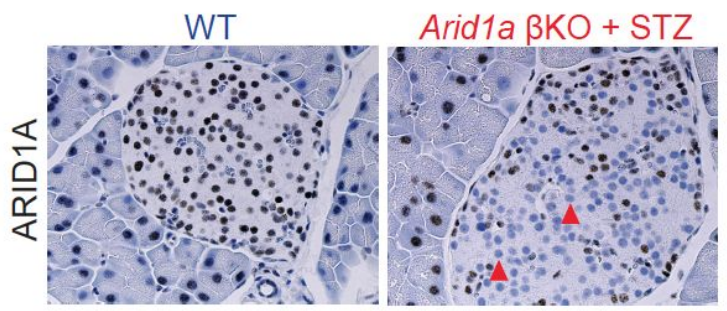

E

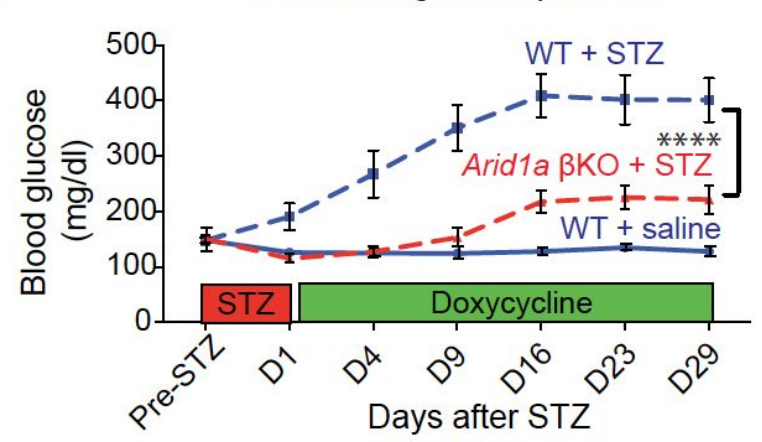

B
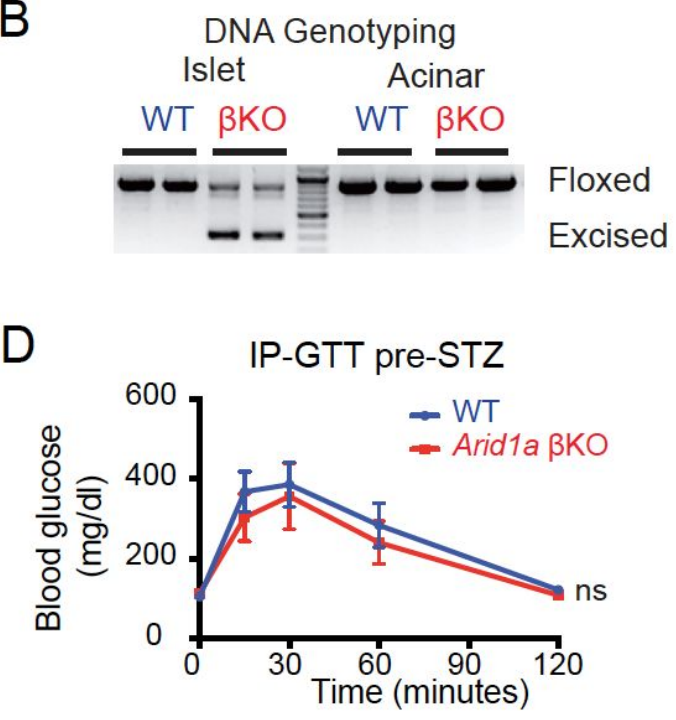

F

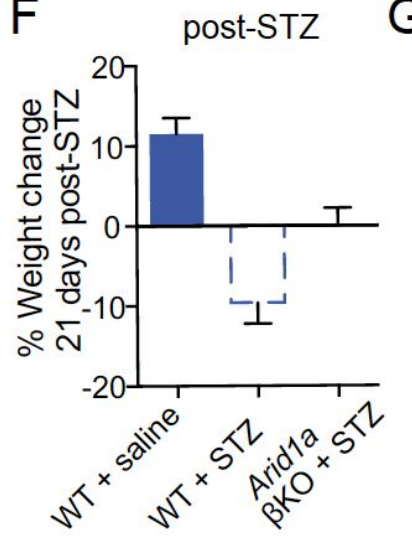

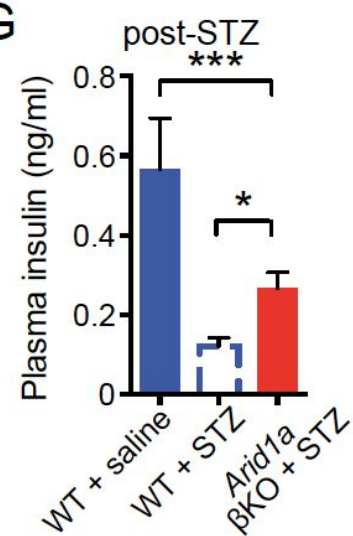

Figure 3 
Figure 3. Arid1a deficiency leads to a $\beta$-cell-mediated anti-diabetic phenotype.

A. Dox-inducible KO of Arid1a in mouse $\beta$-cells (Arid1a $\beta \mathrm{KO}$ ). rtTA is expressed under the control of the mouse insulin promoter (MIP). In the presence of doxycycline (Dox), rtTA activates the transcription of the TRE-Cre transgene. CRE in turn converts the floxed Arid1a alleles to knockout alleles. Dox $(1 \mathrm{mg} / \mathrm{mL})$ was added to the drinking water to conditionally induce Arid1a deletion.

B. Genotyping of islets and acinar cells to assess the recombination at the Arid1a ${ }^{\text {FIFI }}$ locus. Partial excision is expected in islets since cell types other than $\beta$ cells are present.

C. Immunostaining of ARID1A in WT and Arid1a $\beta K O$ pancreata.

D. IP glucose tolerance test 2 weeks post-dox.

E. Fed state blood glucose after STZ. 5 doses of $50 \mathrm{mg} / \mathrm{kg}$ STZ were injected before dox-induced $\beta$-cell deletion of Arid1a. After the last dose of STZ, dox (1 mg/mL) was provided in drinking water $(n=4$ mice for $W T+$ saline, $n=12$ mice for $W T+S T Z, n=10$ mice for Arid1a $\beta K O+S T Z)$.

F. $\%$ body weight change 21 days post-STZ.

G. Plasma insulin levels measured by ELISA 30 days post-STZ. 


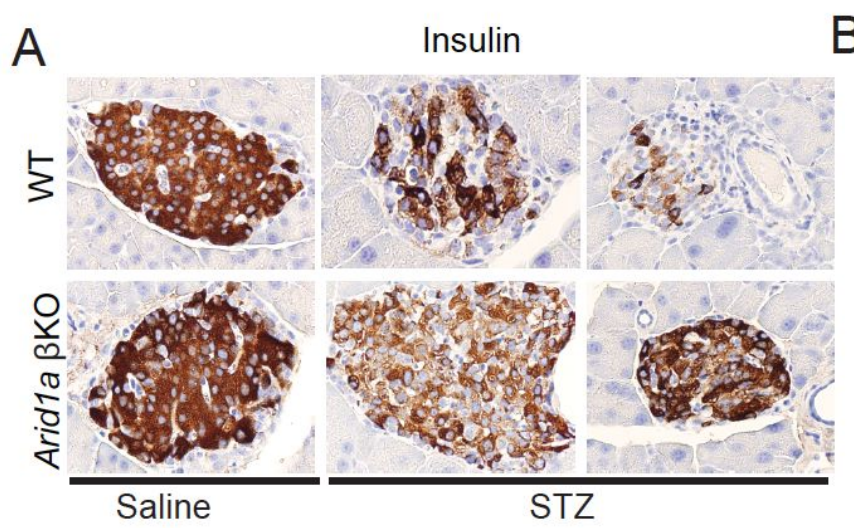

B Glucagon

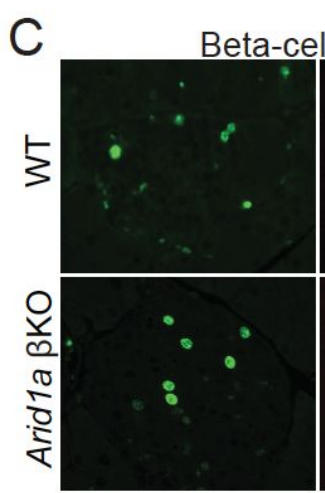

Ki-67

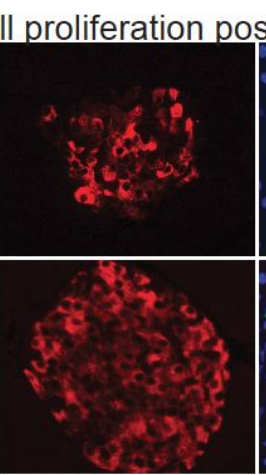

Insulin
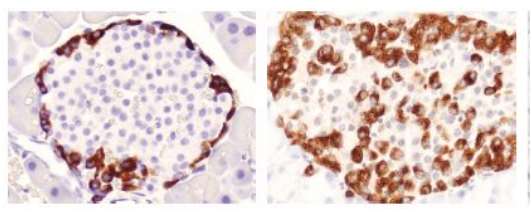

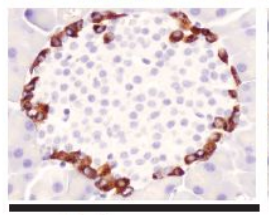

Saline

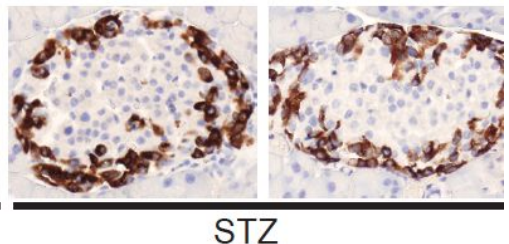

Post STZ

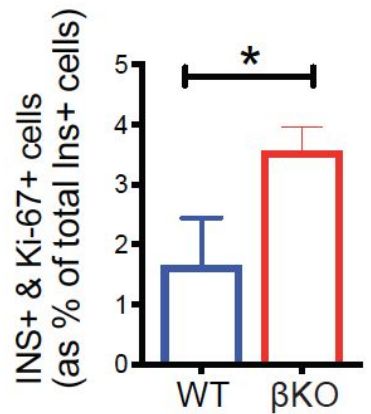

G Post PPx

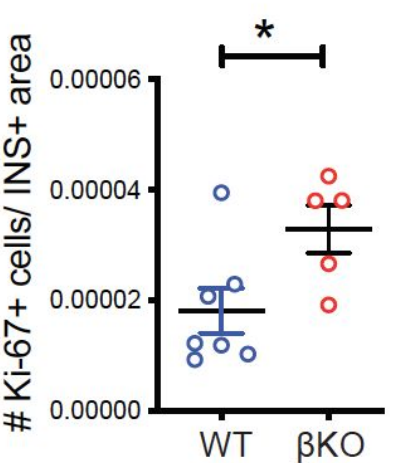

Figure 4 
Figure 4. Arid1a loss results in increased $\beta$-cell survival and proliferation.

A. Insulin staining of WT and Arid1a $\beta$ KO pancreata treated with saline or STZ. Samples were collected 1 month post-STZ.

B. Glucagon staining of WT and Arid1a $\beta K O$ pancreata treated with saline or STZ. Samples were collected 1 month post-STZ.

C. Representative Ki-67 staining (green) of WT and Arid1a $\beta K O$ pancreata post-STZ. Insulin is used as a $\beta$-cell marker (red). Samples were collected 1 month post-STZ.

D. Quantification of the percentage of insulin+ \& Ki-67+ cells to all insulin+ cells (total of 9 islets from 1 WT and total of 25 islets from 3 Arid1a $\beta K O$ mice)

E. The number of islets per pancreas section area in WT and Arid1a $\beta K O$ mice 3 weeks post-STZ. Each dot represents the total number of islets per section area in each mouse ( $\mathrm{n}=4 \mathrm{WT}$ mice for WT and $\mathrm{n}=6$ Arid1a $\beta K O$ mice).

F. Individual islet area $(\mathrm{n}=4$ WT mice and 6 Arid1a $\beta K O$ mice, at least 10 islets were measured for each mouse).

G. Ki-67+ cell number/insulin+ area in islets from regenerated pancreata 6 days after PPx ( $\mathrm{n}=7 \mathrm{WT}$ and 5 Arid1a $\beta \mathrm{KO}$ mice, values from $\sim 10$ islets per mice were calculated and averaged to get a single data point in the graph). 
bioRxiv preprint doi: https://doi.org/10.1101/2020.02.10.942615; this version posted February 11, 2020. The copyright holder for this preprint (which was not certified by peer review) is the author/funder, who has granted bioRxiv a license to display the preprint in perpetuity. It is made available under aCC-BY-NC-ND 4.0 International license.

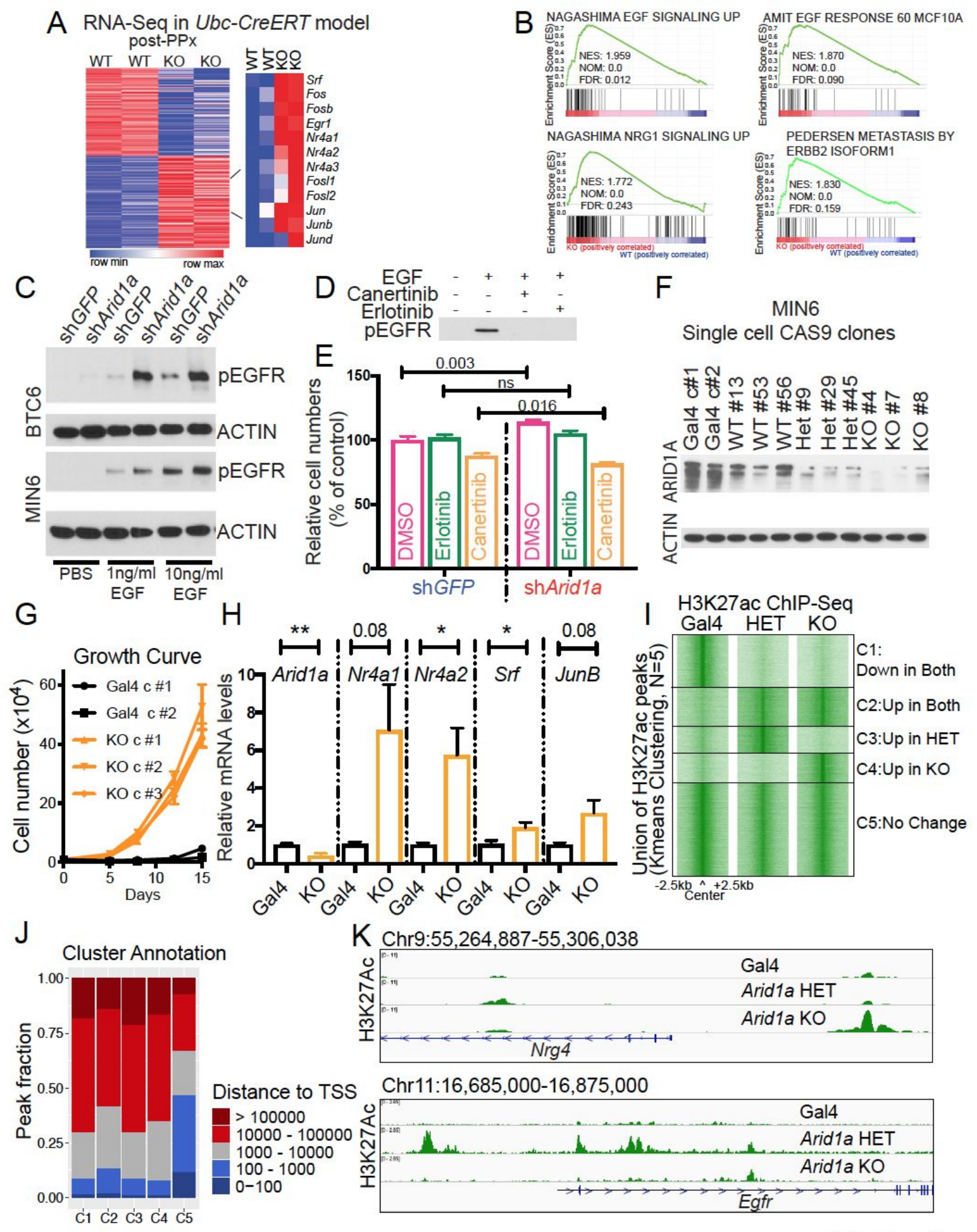

Figure 5 
Figure 5. Arid1a deficient $\beta$-cells have a dependence on increased EGF/Neuregulin signaling.

A. Heatmap of differentially expressed genes in WT and KO islets from Arid1a ${ }^{F / F I}$ and Ubc-CreER; Arid1a ${ }^{\text {FIFI }}$ mice, isolated after PPx and detected by RNA-Seq (left). Heatmap showing a subset of overexpressed genes in $\mathrm{KO}$ islets (right; $\mathrm{n}=2$ and 2 mice).

B. GSEA shows that EGF and NRG1 response genes are upregulated in KO islets. The nominal enrichment score (NES), nominal p-value, and false discovery rate (FDR) q-value are shown within each GSEA plot.

C. p-EGFR western blots in MIN6 and BTC6 treated with different doses of EGF.

D. p-EGFR western blots in WT MIN6 cells treated with EGF in the presence or absence of erlotinib and canertinib.

E. Relative cell numbers for control shGFP and shArid1a MIN6 cells in the presence of canertinib and erlotinib. Shown as \% of control cells (shGFP group treated with DMSO).

F. Western blot showing ARID1A levels in MIN6 clones. MIN6 cells were transduced with lenti-CAS9-blasticidin and either non-targeting lenti-sgRNA (Gal4)-puromycin or lenti-sgRNA (Arid1a)-puromycin.

G. MIN6 clone growth over 15 days measured by cell counting.

H. mRNA expression of selected genes in Gal4 control and Arid1a KO MIN6 clones as measured by qPCR.

I. K-mean clustering of H3K27Ac ChIP-Seq peaks in non-targeting Gal4, Arid1a heterozygous and Arid1a KO MIN6 clones.

J. Annotation of clusters defined by H3K27Ac ChIP-Seq sites by distance to TSS.

K. Sample tracks for H3K27Ac ChIP-Seq in control, Arid1a heterozygous and Arid1a KO MIN6 single cell clones at the Nrg4 and Egfr loci. 
A SWI/SNF binding in ERBB3 regulatory regions

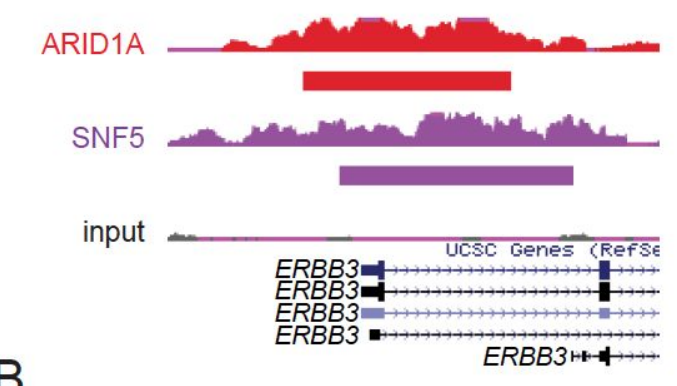

Inducible beta-cell specific ERBB3 overexpression

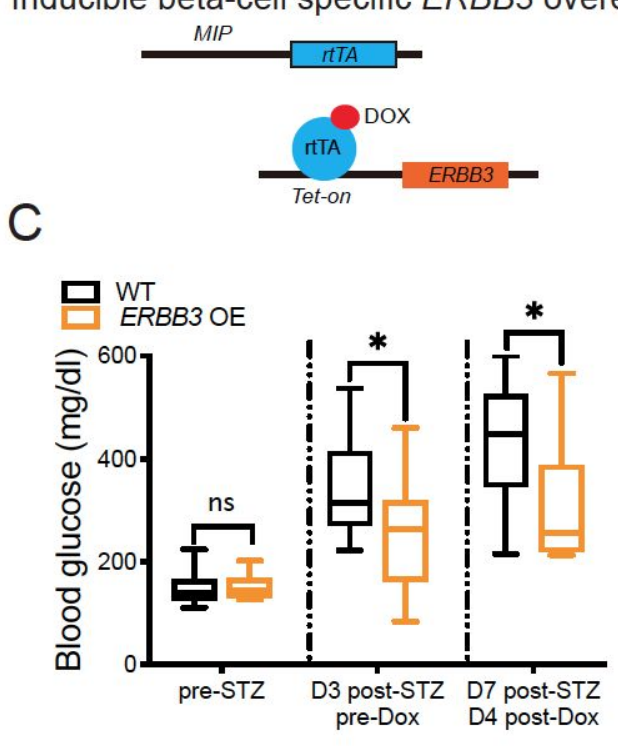

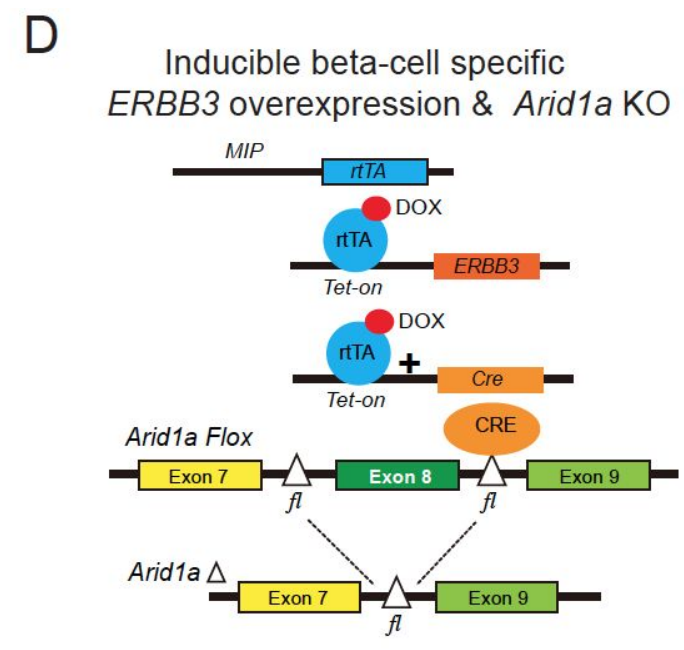

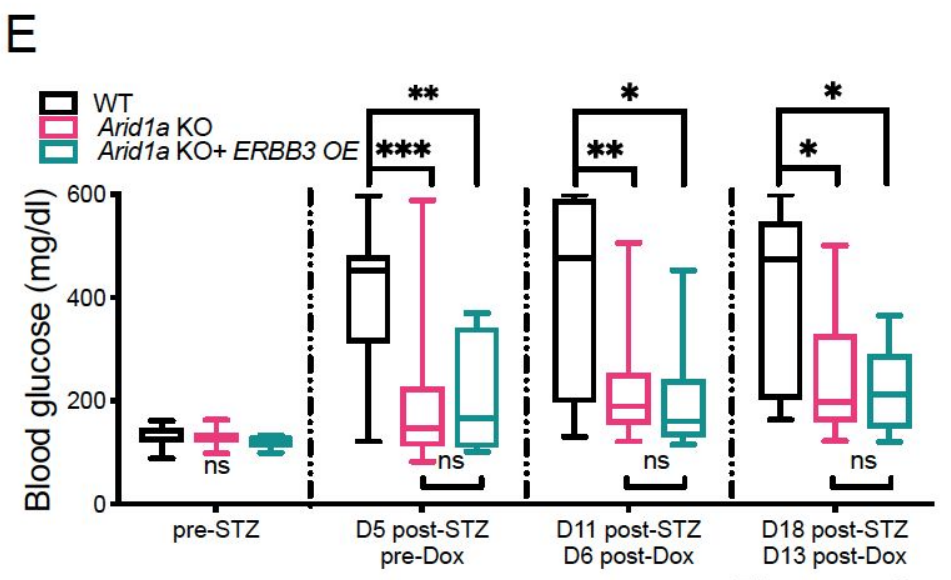

Figure 6

Figure 6. ERBB3 overexpression in $\beta$-cells is protective against T1D after STZ.

A. Binding of ARID1A and SNF5, a core SWI/SNF subunit, to the ERBB3 locus in HepG2 cells (ChIP-seq data from (Raab et al., 2015)).

B. Schema of dox-inducible $\beta$-cell specific ERBB3 overexpression mice: MIP-rtTA; TRE-ERBB3.

C. Fed state blood glucose measurements in control and ERBB3 overexpressing mice post-STZ.

D. Schema of dox-inducible $\beta$-cell specific ERBB3 overexpression and Arid1a $\beta \mathrm{KO}$ mice: MIP-rtTA; TRE-ERBB3; TRE-Cre; Arid1a ${ }^{f / f t}$.

E. Fed state blood glucose measurements in WT, Arid1a $\beta \mathrm{KO}$ and Arid1a $\beta \mathrm{KO}+$ ERBB3 OE mice post-STZ. 

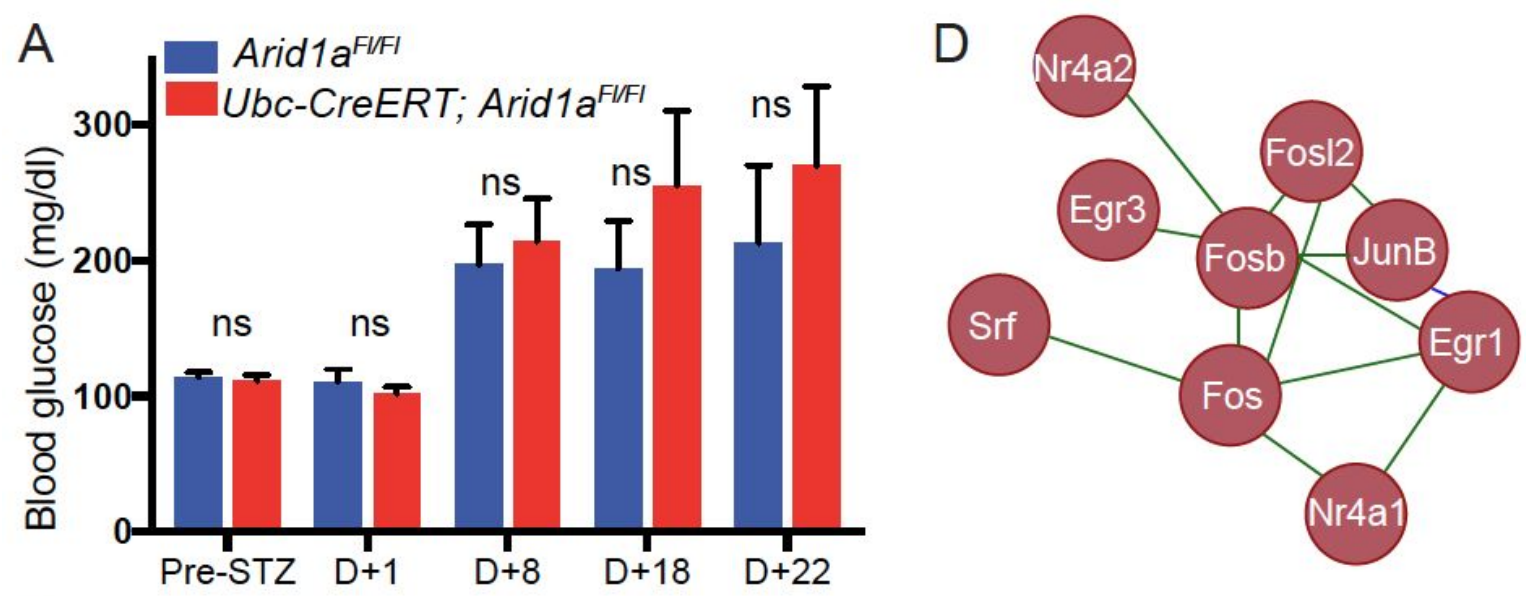

B

Pre-PPx

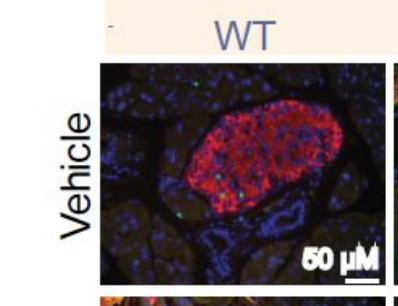

\section{Arid1a $\beta$ KO}

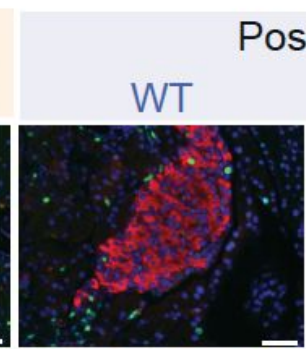

Post-PPx
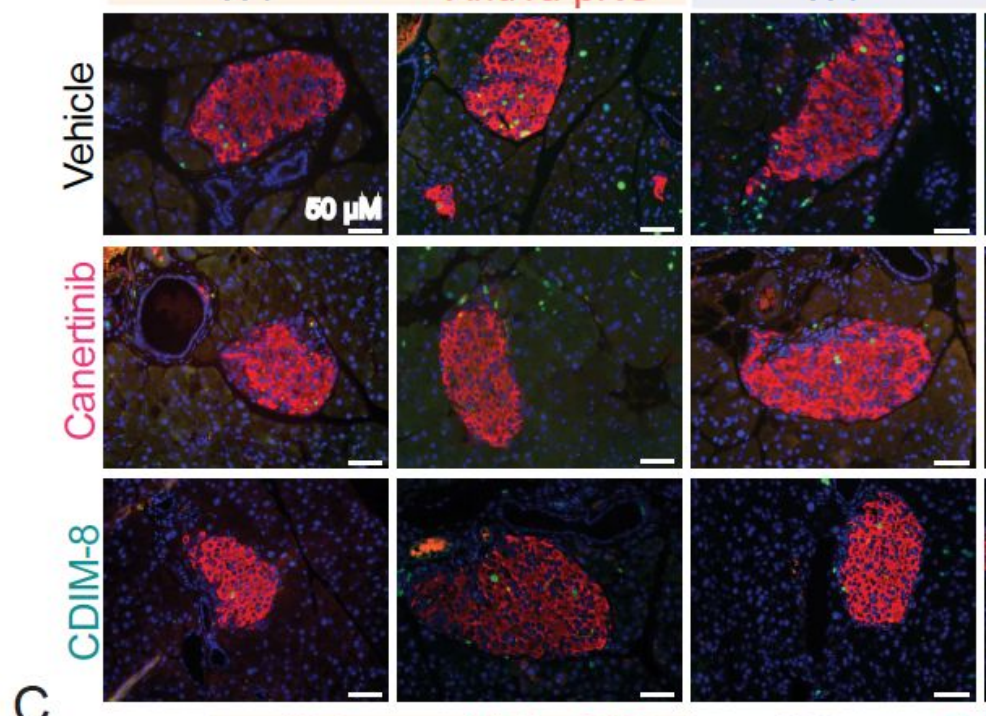

Arid1a $\beta K O$
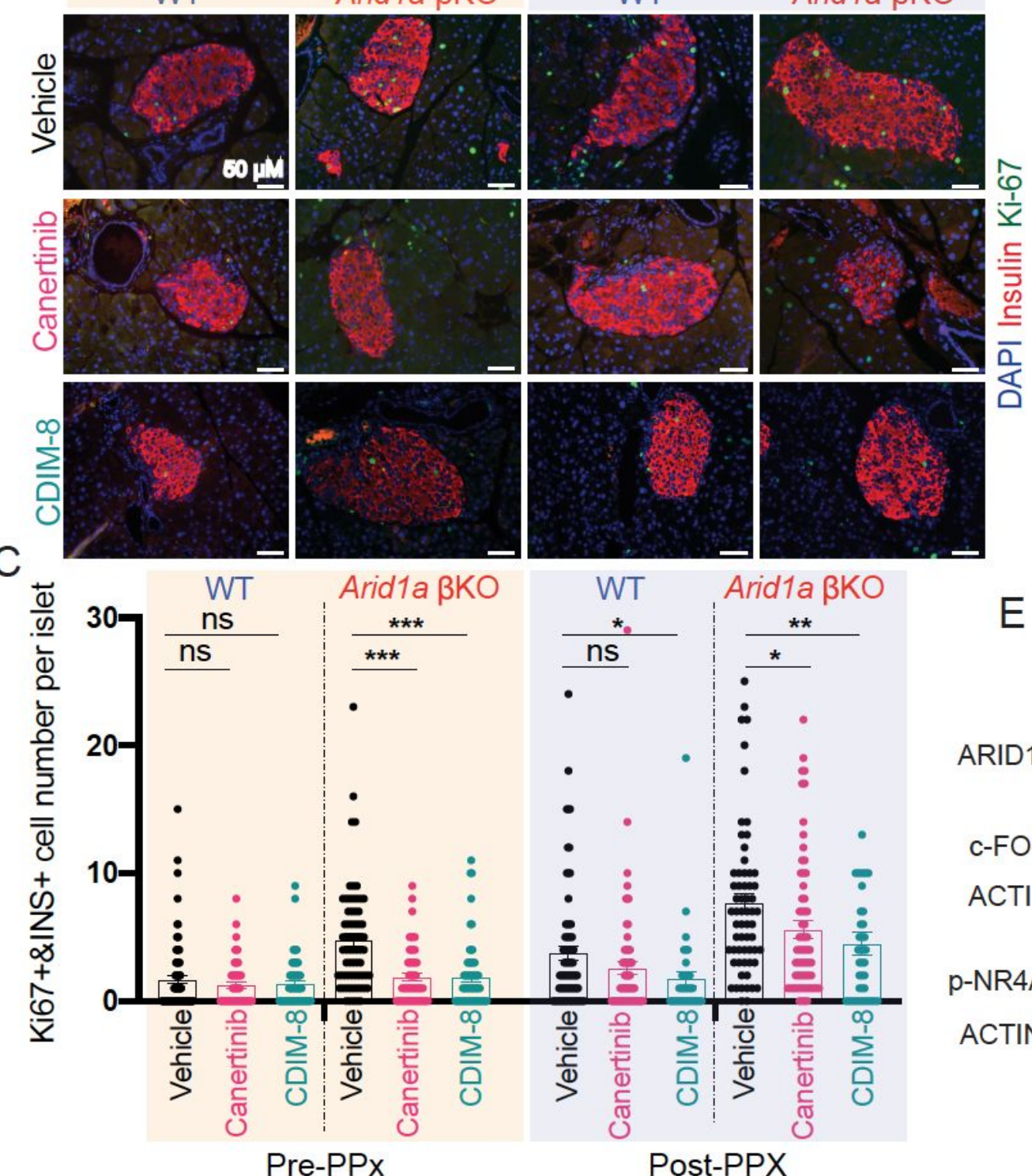

E Mouse islets Post-PPx
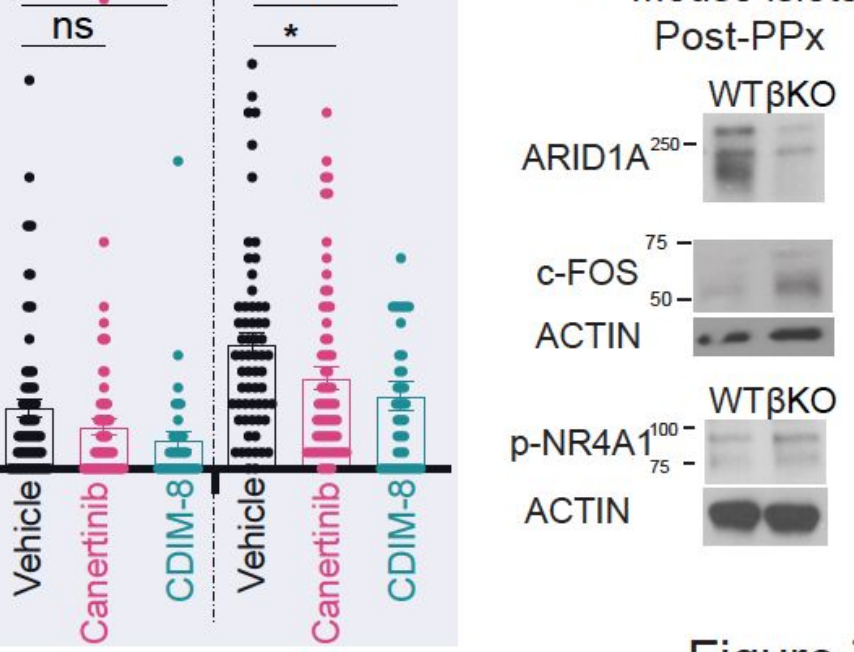

Post-PPX
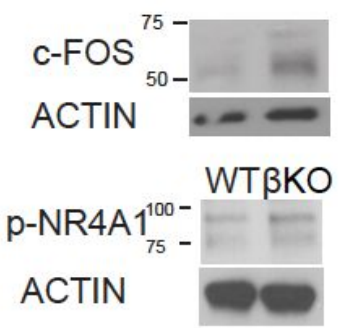

Figure 7 
Figure 7. Pan-ERBB and NR4A1 inhibition abrogated the Arid1a KO phenotype in vivo.

A. Fed state blood glucose measurements following STZ in WT and whole body Arid1a KO mice. $20 \mathrm{mg} / \mathrm{kg}$ canertinib was administered daily through oral gavage. Compare to Fig. $2 \mathrm{~F}$, which did not include canertinib.

B. Immunofluorescence for DAPI (blue), insulin (red), and Ki-67 (green) in WT and Arid1a $\beta K O$ islets before or after PPx in the presence of vehicle, canertinib, or CDIM-8 treatment.

C. Quantification of Ki-67 and insulin double positive cell number per islet. Each dot represents an islet. Between 37-75 islets per genotype were counted from 5 to 8 mice per genotype.

D. The STRING database predicts an important protein-protein interaction network from differentially overexpressed genes found in RNA-seq data.

E. Western blot analysis of c-FOS and p-NR4A1 in mouse islets isolated 6 day post-PPx. 

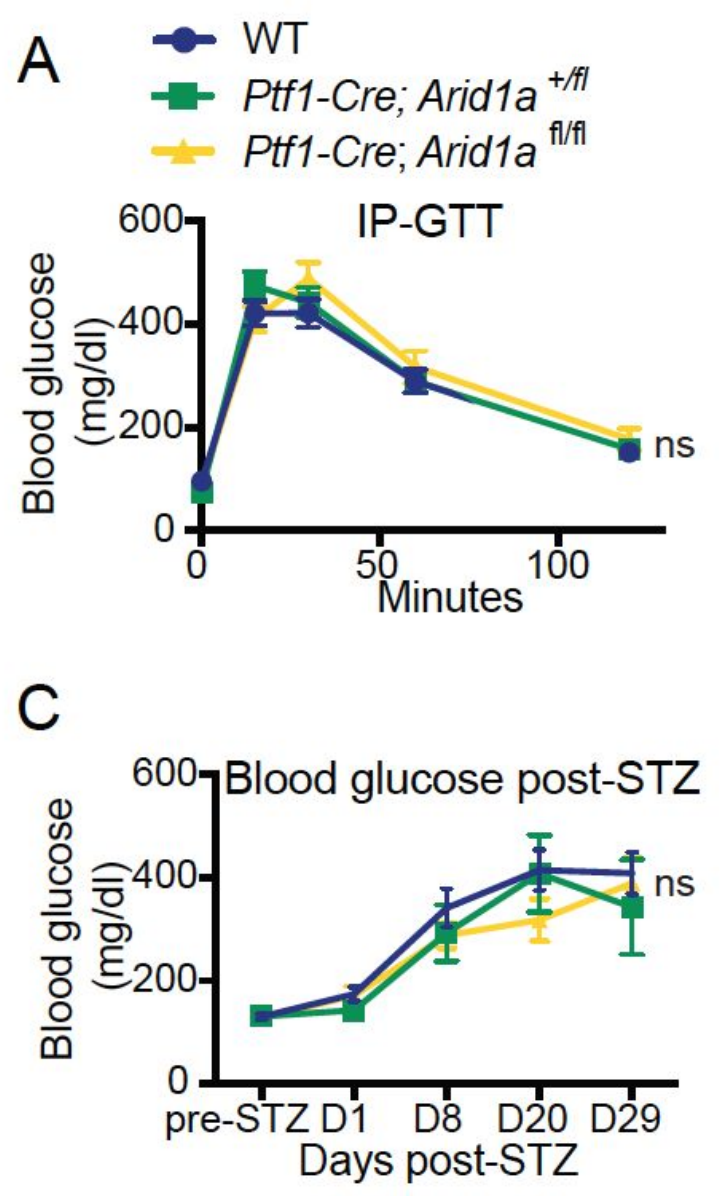

B

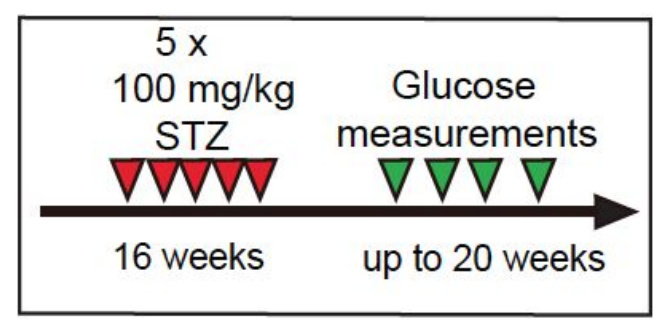

\section{Figure S1}

Figure S1. Acinar and ductal knockout of Arid1a does not phenocopy the whole body knockout.

A. Intraperitoneal glucose tolerance test for Arid1a $a^{F / F I}$, Ptf1a-Cre; Arid1a ${ }^{+/ F I}$, and Ptf1a-Cre; Arid1a $a^{F I / F I}$ mice after overnight fasting.

B. Experimental timeline for STZ-induced diabetes.

C. Fed state blood glucose measurements taken post-STZ. 
bioRxiv preprint doi: https://doi.org/10.1101/2020 02 10.942615; this version posted February 11, 2020. The copyright holder for this preprint (which was not certified by peer review) is the author/funder, who has granted bioRxiv a license to display the preprint in perpetuity. It is made available under aCC-BY-NC-ND 4.0 International license.

A

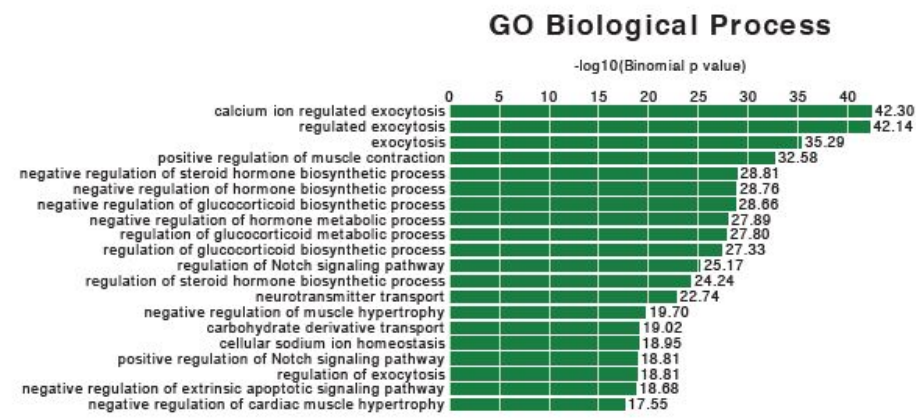

\section{B Cis-Regulatory Function Analysis by GREAT on Cluster 4}
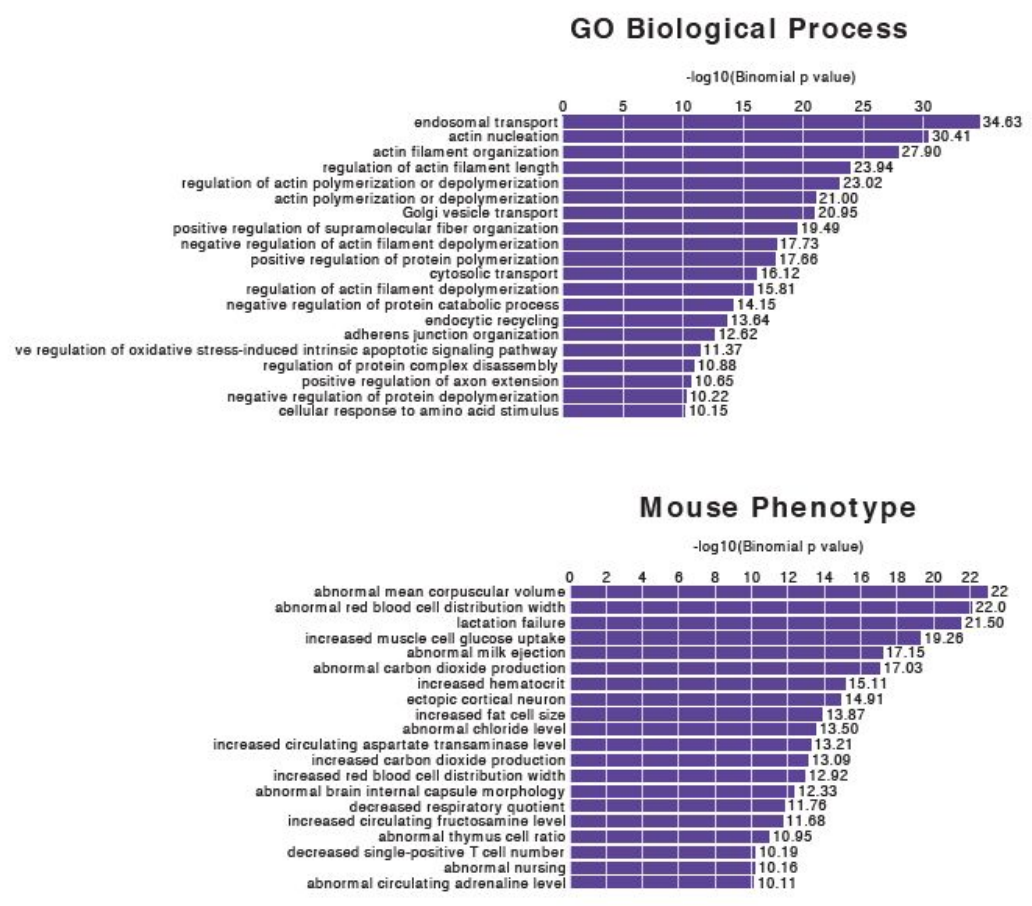

Figure S2 
Figure S2. Cis-regulatory function analysis by GREAT for H3K27Ac peaks.

A. Cis-regulatory function analysis by GREAT on sites within Cluster 3 (increased H3K27Ac in Arid1a HET condition) given as GO processes and mouse phenotypes.

B. Cis-regulatory function analysis by GREAT on sites within Cluster 4 (increased H3K27Ac in Arid1a KO condition) given as GO processes and mouse phenotypes. 


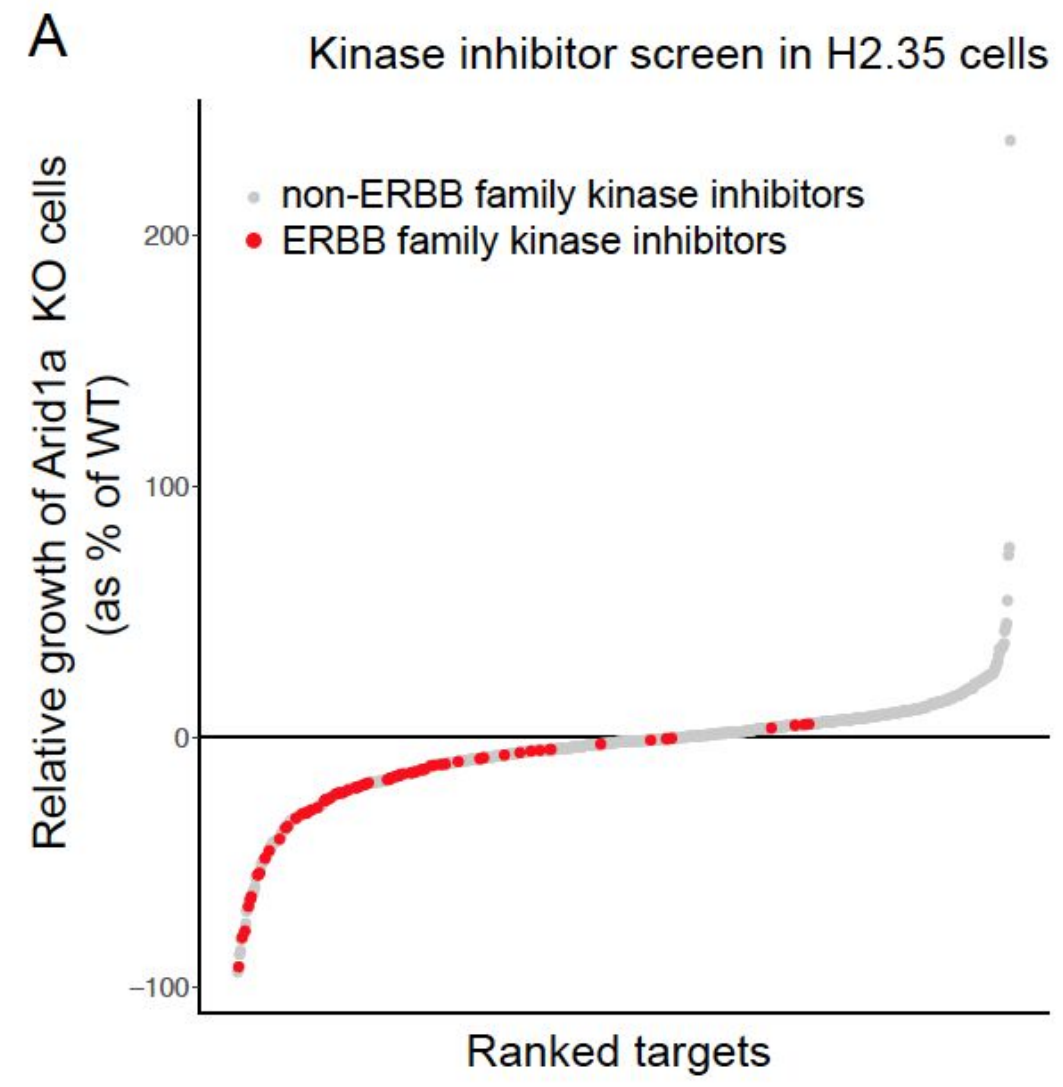

B

Top 10 inhibitors causing synthetic lethality in Arid1a KO cells

\begin{tabular}{|l|c|c|}
\hline Inhibitor name & Target kinase & Relative growth \\
\hline PHA-665752 & C-MET & $-94 \%$ \\
\hline Afatinib (BIBW2992) & EGFR, ERBB2 & $-92 \%$ \\
\hline Ponatinib (AP24534) & BCR-ABL & $-87 \%$ \\
\hline R935788 & SYK & $-85 \%$ \\
\hline R406 & SYK & $-81 \%$ \\
\hline WZ8040 & EGFR & $-80 \%$ \\
\hline LDN193189 & ALK1, ALK2, ALK3, ALK6 & $-79 \%$ \\
\hline R788 (Fostamatinib) & SYK & $-78 \%$ \\
\hline Cl-1033 (Canertinib) & EGFR, ERBB2, ERBB4 & $-77 \%$ \\
\hline YM201636 & PIP5KIII & $-75 \%$ \\
\hline
\end{tabular}

Figure S3 
Figure S3. Kinase inhibitor screen in H2.35 cells shows that Arid1a deficient cells are more sensitive to cell growth inhibition induced by ERBB inhibitors than WT cells.

A. Relative cell growth (KO vs WT). About 300 kinase inhibitors were used in this screen. Each gray dot corresponds to relative growth of Arid1a KO vs. WT cells in the presence of a non-ERBB kinase inhibitor, whereas each red dot corresponds to an ERBB family kinase inhibitor.

B. Table showing the top 10 kinase inhibitors that caused the highest reduction of growth/viability selectively in Arid1a KO cells. 3 out of 10 top kinase inhibitors targeted the ERBB family of RTKs.

\section{REFERENCES}

Ackermann Misfeldt, A., Costa, R.H., and Gannon, M. (2008). Beta-cell proliferation, but not neogenesis, following $60 \%$ partial pancreatectomy is impaired in the absence of FoxM1. Diabetes 57, 3069-3077.

Aguayo-Mazzucato, C., and Bonner-Weir, S. (2018). Pancreatic $\beta$ cell regeneration as a possible therapy for diabetes. Cell Metab. 27, 57-67.

Alismail, H., and Jin, S. (2014). Microenvironmental stimuli for proliferation of functional islet $\beta$-cells. Cell Biosci. 4, 12.

Alver, B.H., Kim, K.H., Lu, P., Wang, X., Manchester, H.E., Wang, W., Haswell, J.R., Park, P.J., and Roberts, C.W.M. (2017). The SWI/SNF chromatin remodelling complex is required for maintenance of lineage specific enhancers. Nat. Commun. 8, 14648.

Argiras, E.P., Blakeley, C.R., Dunnill, M.S., Otremski, S., and Sykes, M.K. (1987). High PEEP decreases hyaline membrane formation in surfactant deficient lungs. Br. J. Anaesth. 59, 1278-1285.

Barrett, J.C., Clayton, D.G., Concannon, P., Akolkar, B., Cooper, J.D., Erlich, H.A., Julier, C., Morahan, G., Nerup, J., Nierras, C., et al. (2009). Genome-wide association study and meta-analysis find that over 40 loci affect risk of type 1 diabetes. Nat. Genet. 41, 703-707.

Bradfield, J.P., Qu, H.-Q., Wang, K., Zhang, H., Sleiman, P.M., Kim, C.E., Mentch, F.D., Qiu, H., Glessner, J.T., Thomas, K.A., et al. (2011). A genome-wide meta-analysis of six type 1 diabetes cohorts identifies multiple associated loci. PLoS Genet. 7, e1002293.

Celen, C., Chuang, J.-C., Luo, X., Nijem, N., Walker, A.K., Chen, F., Zhang, S., Chung, A.S., 
Nguyen, L.H., Nassour, I., et al. (2017). Arid1b haploinsufficient mice reveal neuropsychiatric phenotypes and reversible causes of growth impairment. Elife 6.

Chandler, R.L., Brennan, J., Schisler, J.C., Serber, D., Patterson, C., and Magnuson, T. (2013). ARID1a-DNA interactions are required for promoter occupancy by SWI/SNF. Mol. Cell. Biol. 33, 265-280.

De León, D.D., Deng, S., Madani, R., Ahima, R.S., Drucker, D.J., and Stoffers, D.A. (2003). Role of endogenous glucagon-like peptide-1 in islet regeneration after partial pancreatectomy. Diabetes 52, 365-371.

Dor, Y., Brown, J., Martinez, O.I., and Melton, D.A. (2004). Adult pancreatic beta-cells are formed by self-duplication rather than stem-cell differentiation. Nature 429, 41-46.

D'Uva, G., Aharonov, A., Lauriola, M., Kain, D., Yahalom-Ronen, Y., Carvalho, S., Weisinger, K., Bassat, E., Rajchman, D., Yifa, O., et al. (2015). ERBB2 triggers mammalian heart regeneration by promoting cardiomyocyte dedifferentiation and proliferation. Nat. Cell Biol. 17, $627-638$.

Gaddy, D.F., Riedel, M.J., Pejawar-Gaddy, S., Kieffer, T.J., and Robbins, P.D. (2010). In vivo expression of HGF/NK1 and GLP-1 From dsAAV vectors enhances pancreatic ß-cell proliferation and improves pathology in the $\mathrm{db} / \mathrm{db}$ mouse model of diabetes. Diabetes 59 , 3108-3116.

Gao, X., Tate, P., Hu, P., Tjian, R., Skarnes, W.C., and Wang, Z. (2008). ES cell pluripotency and germ-layer formation require the SWI/SNF chromatin remodeling component BAF250a. Proc Natl Acad Sci USA 105, 6656-6661.

Gehrke, A.R., Neverett, E., Luo, Y.-J., Brandt, A., Ricci, L., Hulett, R.E., Gompers, A., Ruby, J.G., Rokhsar, D.S., Reddien, P.W., et al. (2019). Acoel genome reveals the regulatory landscape of whole-body regeneration. Science 363.

Gemberling, M., Karra, R., Dickson, A.L., and Poss, K.D. (2015). Nrg1 is an injury-induced cardiomyocyte mitogen for the endogenous heart regeneration program in zebrafish. Elife 4.

Hajmrle, C., Smith, N., Spigelman, A.F., Dai, X., Senior, L., Bautista, A., Ferdaoussi, M., and MacDonald, P.E. (2016). Interleukin-1 signaling contributes to acute islet compensation. JCI Insight 1, e86055.

Han, L., Madan, V., Mayakonda, A., Dakle, P., Woon, T.W., Shyamsunder, P., Nordin, H.B.M., Cao, Z., Sundaresan, J., Lei, I., et al. (2019). Chromatin remodeling mediated by ARID1A is indispensable for normal hematopoiesis in mice. Leukemia 33, 2291-2305.

Harrow, J., Frankish, A., Gonzalez, J.M., Tapanari, E., Diekhans, M., Kokocinski, F., Aken, B.L., Barrell, D., Zadissa, A., Searle, S., et al. (2012). GENCODE: the reference human genome annotation for The ENCODE Project. Genome Res. 22, 1760-1774.

Hota, S.K., Johnson, J.R., Verschueren, E., Thomas, R., Blotnick, A.M., Zhu, Y., Sun, X., Pennacchio, L.A., Krogan, N.J., and Bruneau, B.G. (2019). Dynamic BAF chromatin remodeling complex subunit inclusion promotes temporally distinct gene expression programs in 
cardiogenesis. Development 146.

Jopling, C., Boue, S., and Izpisua Belmonte, J.C. (2011). Dedifferentiation, transdifferentiation and reprogramming: three routes to regeneration. Nat. Rev. Mol. Cell Biol. 12, 79-89.

Kaur, S., Mirza, A.H., Brorsson, C.A., Fløyel, T., Størling, J., Mortensen, H.B., Pociot, F., and Hvidoere International Study Group (2016). The genetic and regulatory architecture of ERBB3-type 1 diabetes susceptibility locus. Mol. Cell. Endocrinol. 419, 83-91.

Kawaguchi, Y., Cooper, B., Gannon, M., Ray, M., MacDonald, R.J., and Wright, C.V.E. (2002). The role of the transcriptional regulator Ptf1a in converting intestinal to pancreatic progenitors. Nat. Genet. 32, 128-134.

Kelso, T.W.R., Porter, D.K., Amaral, M.L., Shokhirev, M.N., Benner, C., and Hargreaves, D.C. (2017). Chromatin accessibility underlies synthetic lethality of SWI/SNF subunits in ARID1A-mutant cancers. Elife 6.

Kim, D., Langmead, B., and Salzberg, S.L. (2015). HISAT: a fast spliced aligner with low memory requirements. Nat. Methods 12, 357-360.

Kusminski, C.M., Chen, S., Ye, R., Sun, K., Wang, Q.A., Spurgin, S.B., Sanders, P.E., Brozinick, J.T., Geldenhuys, W.J., Li, W.-H., et al. (2016). MitoNEET-Parkin Effects in Pancreatic $\alpha$ - and $\beta$-Cells, Cellular Survival, and Intrainsular Cross Talk. Diabetes 65, 1534-1555.

Langmead, B., and Salzberg, S.L. (2012). Fast gapped-read alignment with Bowtie 2. Nat. Methods 9, 357-359.

Liao, Y., Smyth, G.K., and Shi, W. (2014). featureCounts: an efficient general purpose program for assigning sequence reads to genomic features. Bioinformatics 30, 923-930.

Li, H., Handsaker, B., Wysoker, A., Fennell, T., Ruan, J., Homer, N., Marth, G., Abecasis, G., Durbin, R., and 1000 Genome Project Data Processing Subgroup (2009). The Sequence Alignment/Map format and SAMtools. Bioinformatics 25, 2078-2079.

Martín, F., Andreu, E., Rovira, J.M., Pertusa, J.A., Raurell, M., Ripoll, C., Sanchez-Andrés, J.V., Montanya, E., and Soria, B. (1999). Mechanisms of glucose hypersensitivity in beta-cells from normoglycemic, partially pancreatectomized mice. Diabetes 48, 1954-1961.

Mashtalir, N., D’Avino, A.R., Michel, B.C., Luo, J., Pan, J., Otto, J.E., Zullow, H.J., McKenzie, Z.M., Kubiak, R.L., St Pierre, R., et al. (2018). Modular organization and assembly of SWI/SNF family chromatin remodeling complexes. Cell 175, 1272-1288.e20.

Mathur, R., Alver, B.H., San Roman, A.K., Wilson, B.G., Wang, X., Agoston, A.T., Park, P.J., Shivdasani, R.A., and Roberts, C.W.M. (2017). ARID1A loss impairs enhancer-mediated gene regulation and drives colon cancer in mice. Nat. Genet. 49, 296-302.

McCarthy, D.J., Chen, Y., and Smyth, G.K. (2012). Differential expression analysis of multifactor RNA-Seq experiments with respect to biological variation. Nucleic Acids Res. 40, 4288-4297.

McKenna, B., Guo, M., Reynolds, A., Hara, M., and Stein, R. (2015). Dynamic recruitment of 
functionally distinct Swi/Snf chromatin remodeling complexes modulates $\mathrm{Pdx} 1$ activity in islet $\beta$ cells. Cell Rep. 10, 2032-2042.

Meier, J.J., Butler, A.E., Saisho, Y., Monchamp, T., Galasso, R., Bhushan, A., Rizza, R.A., and Butler, P.C. (2008). Beta-cell replication is the primary mechanism subserving the postnatal expansion of beta-cell mass in humans. Diabetes 57, 1584-1594.

Miettinen, P.J., Ustinov, J., Ormio, P., Gao, R., Palgi, J., Hakonen, E., Juntti-Berggren, L., Berggren, P.-O., and Otonkoski, T. (2006). Downregulation of EGF receptor signaling in pancreatic islets causes diabetes due to impaired postnatal beta-cell growth. Diabetes 55 , 3299-3308.

Mina, M., Magi, S., Jurman, G., Itoh, M., Kawaji, H., Lassmann, T., Arner, E., Forrest, A.R.R., Carninci, P., Hayashizaki, Y., et al. (2015). Promoter-level expression clustering identifies time development of transcriptional regulatory cascades initiated by ErbB receptors in breast cancer cells. Sci. Rep. 5, 11999.

Nakayama, R.T., Pulice, J.L., Valencia, A.M., McBride, M.J., McKenzie, Z.M., Gillespie, M.A., Ku, W.L., Teng, M., Cui, K., Williams, R.T., et al. (2017). SMARCB1 is required for widespread BAF complex-mediated activation of enhancers and bivalent promoters. Nat. Genet. 49, 1613-1623.

Nikitin, A.G., Lavrikova, E.I., Seregin, I.A., Zil'berman, L.I., Tsitlidze, N.M., Kuraeva, T.L., Peterkova, V.A., Dedov, I.I., and Nosikov, V.V. (2010). [Association of the polymorphisms of the ERBB3 and SH2B3 genes with type 1 diabetes]. Mol Biol (Mosk) 44, 257-262.

Oh, Y.S., Shin, S., Lee, Y.-J., Kim, E.H., and Jun, H.-S. (2011). Betacellulin-induced beta cell proliferation and regeneration is mediated by activation of ErbB-1 and ErbB-2 receptors. PLoS ONE 6, e23894.

Pan, J., McKenzie, Z.M., D’Avino, A.R., Mashtalir, N., Lareau, C.A., St Pierre, R., Wang, L., Shilatifard, A., and Kadoch, C. (2019). The ATPase module of mammalian SWI/SNF family complexes mediates subcomplex identity and catalytic activity-independent genomic targeting. Nat. Genet. 51, 618-626.

Perl, A.-K.T., Wert, S.E., Nagy, A., Lobe, C.G., and Whitsett, J.A. (2002). Early restriction of peripheral and proximal cell lineages during formation of the lung. Proc Natl Acad Sci USA 99, 10482-10487.

Peshavaria, M., Larmie, B.L., Lausier, J., Satish, B., Habibovic, A., Roskens, V., Larock, K., Everill, B., Leahy, J.L., and Jetton, T.L. (2006). Regulation of pancreatic beta-cell regeneration in the normoglycemic 60\% partial-pancreatectomy mouse. Diabetes 55, 3289-3298.

Porat, S., Weinberg-Corem, N., Tornovsky-Babaey, S., Schyr-Ben-Haroush, R., Hija, A., Stolovich-Rain, M., Dadon, D., Granot, Z., Ben-Hur, V., White, P., et al. (2011). Control of pancreatic $\beta$ cell regeneration by glucose metabolism. Cell Metab. 13, 440-449.

Quinlan, A.R., and Hall, I.M. (2010). BEDTools: a flexible suite of utilities for comparing genomic features. Bioinformatics 26, 841-842. 
Raab, J.R., Resnick, S., and Magnuson, T. (2015). Genome-Wide Transcriptional Regulation Mediated by Biochemically Distinct SWI/SNF Complexes. PLoS Genet. 11, e1005748.

Rieck, S., and Kaestner, K.H. (2010). Expansion of beta-cell mass in response to pregnancy. Trends Endocrinol. Metab. 21, 151-158.

Robinson, M.D., McCarthy, D.J., and Smyth, G.K. (2010). edgeR: a Bioconductor package for differential expression analysis of digital gene expression data. Bioinformatics 26, 139-140.

Safe, S., Jin, U.-H., Morpurgo, B., Abudayyeh, A., Singh, M., and Tjalkens, R.B. (2016). Nuclear receptor 4A (NR4A) family - orphans no more. J. Steroid Biochem. Mol. Biol. 157, 48-60.

Shen, L., Shao, N., Liu, X., and Nestler, E. (2014). ngs.plot: Quick mining and visualization of next-generation sequencing data by integrating genomic databases. BMC Genomics 15, 284.

Song, Z., Fusco, J., Zimmerman, R., Fischbach, S., Chen, C., Ricks, D.M., Prasadan, K., Shiota, C., Xiao, X., and Gittes, G.K. (2016). Epidermal growth factor receptor signaling regulates $\beta$ cell proliferation in adult mice. J. Biol. Chem. 291, 22630-22637.

Spaeth, J.M., Liu, J.-H., Peters, D., Guo, M., Osipovich, A.B., Mohammadi, F., Roy, N., Bhushan, A., Magnuson, M.A., Hebrok, M., et al. (2019). The Pdx1-Bound Swi/Snf Chromatin Remodeling Complex Regulates Pancreatic Progenitor Cell Proliferation and Mature Islet $\beta$-Cell Function. Diabetes 68, 1806-1818.

Sun, C., Wei, H., Chen, X., Zhao, Z., Du, H., Song, W., Yang, Y., Zhang, M., Lu, W., Pei, Z., et al. (2016a). ERBB3-rs2292239 as primary type 1 diabetes association locus among non-HLA genes in Chinese. Meta Gene 9, 120-123.

Sun, X., Chuang, J.-C., Kanchwala, M., Wu, L., Celen, C., Li, L., Liang, H., Zhang, S., Maples, T., Nguyen, L.H., et al. (2016b). Suppression of the SWI/SNF component arid1a promotes mammalian regeneration. Cell Stem Cell 18, 456-466.

Sun, X., Wang, S.C., Wei, Y., Luo, X., Jia, Y., Li, L., Gopal, P., Zhu, M., Nassour, I., Chuang, J.-C., et al. (2018). Arid1a Has Context-Dependent Oncogenic and Tumor Suppressor Functions in Liver Cancer. Cancer Cell 33, 151-152.

Szklarczyk, D., Franceschini, A., Wyder, S., Forslund, K., Heller, D., Huerta-Cepas, J., Simonovic, M., Roth, A., Santos, A., Tsafou, K.P., et al. (2015). STRING v10: protein-protein interaction networks, integrated over the tree of life. Nucleic Acids Res. 43, D447-52.

Todd, J.A., Walker, N.M., Cooper, J.D., Smyth, D.J., Downes, K., Plagnol, V., Bailey, R., Nejentsev, S., Field, S.F., Payne, F., et al. (2007). Robust associations of four new chromosome regions from genome-wide analyses of type 1 diabetes. Nat. Genet. 39, 857-864.

Vierbuchen, T., Ling, E., Cowley, C.J., Couch, C.H., Wang, X., Harmin, D.A., Roberts, C.W.M., and Greenberg, M.E. (2017). AP-1 Transcription Factors and the BAF Complex Mediate Signal-Dependent Enhancer Selection. Mol. Cell 68, 1067-1082.e12.

Wang, S.C., Nassour, I., Xiao, S., Zhang, S., Luo, X., Lee, J., Li, L., Sun, X., Nguyen, L.H., Chuang, J.-C., et al. (2019a). SWI/SNF component ARID1A restrains pancreatic neoplasia 
formation. Gut 68, 1259-1270.

Wang, W., Friedland, S.C., Guo, B., O’Dell, M.R., Alexander, W.B., Whitney-Miller, C.L., Agostini-Vulaj, D., Huber, A.R., Myers, J.R., Ashton, J.M., et al. (2019b). ARID1A, a SWI/SNF subunit, is critical to acinar cell homeostasis and regeneration and is a barrier to transformation and epithelial-mesenchymal transition in the pancreas. Gut 68, 1245-1258.

Wei, Z., Yoshihara, E., He, N., Hah, N., Fan, W., Pinto, A.F.M., Huddy, T., Wang, Y., Ross, B., Estepa, G., et al. (2018). Vitamin D switches BAF complexes to protect $\beta$ cells. Cell 173, 1135-1149.e15.

Wu, J.I., Lessard, J., and Crabtree, G.R. (2009). Understanding the words of chromatin regulation. Cell 136, 200-206.

Yarden, Y., and Sliwkowski, M.X. (2001). Untangling the ErbB signalling network. Nat. Rev. Mol. Cell Biol. 2, 127-137.

Yu, Y., Chen, Y., Kim, B., Wang, H., Zhao, C., He, X., Liu, L., Liu, W., Wu, L.M.N., Mao, M., et al. (2013). Olig2 targets chromatin remodelers to enhancers to initiate oligodendrocyte differentiation. Cell 152, 248-261.

Zeng, C., Mulas, F., Sui, Y., Guan, T., Miller, N., Tan, Y., Liu, F., Jin, W., Carrano, A.C., Huising, M.O., et al. (2017). Pseudotemporal ordering of single cells reveals metabolic control of postnatal $\beta$ cell proliferation. Cell Metab. 25, 1160-1175.e11.

Zhang, W., Chronis, C., Chen, X., Zhang, H., Spalinskas, R., Pardo, M., Chen, L., Wu, G., Zhu, Z., Yu, Y., et al. (2019). The BAF and PRC2 complex subunits dpf2 and eed antagonistically converge on tbx3 to control ESC differentiation. Cell Stem Cell 24, 138-152.e8.

Zhang, Y., Liu, T., Meyer, C.A., Eeckhoute, J., Johnson, D.S., Bernstein, B.E., Nusbaum, C., Myers, R.M., Brown, M., Li, W., et al. (2008). Model-based analysis of ChIP-Seq (MACS). Genome Biol. 9, R137.

Zhang, Z., Cao, M., Chang, C.-W., Wang, C., Shi, X., Zhan, X., Birnbaum, S.G., Bezprozvanny, I., Huber, K.M., and Wu, J.I. (2016). Autism-Associated Chromatin Regulator Brg1/SmarcA4 Is Required for Synapse Development and Myocyte Enhancer Factor 2-Mediated Synapse Remodeling. Mol. Cell. Biol. 36, 70-83.

Zhan, Y., Du, X., Chen, H., Liu, J., Zhao, B., Huang, D., Li, G., Xu, Q., Zhang, M., Weimer, B.C., et al. (2008). Cytosporone B is an agonist for nuclear orphan receptor Nur77. Nat. Chem. Biol. $4,548-556$.

Zmuda, E.J., Powell, C.A., and Hai, T. (2011). A method for murine islet isolation and subcapsular kidney transplantation. J. Vis. Exp. 